\title{
NuSTAR + XMM-Newton monitoring of the neutron star transient AX J1745.6-2901
}

\author{
G. Ponti, ${ }^{1 \star}$ S. Bianchi, ${ }^{2}$ T. Muños-Darias,${ }^{3,4}$ K. Mori,${ }^{5}$ K. De,${ }^{6}$ A. Rau, ${ }^{1}$ B. De Marco, \\ C. Hailey, ${ }^{5}$ J. Tomsick, ${ }^{8}$ K. K. Madsen, ${ }^{9}$ M. Clavel,${ }^{8}$ F. Rahoui, ${ }^{10,11}$ \\ D. V. Lal, ${ }^{12}$ S. Roy ${ }^{12}$ and D. Stern ${ }^{13}$ \\ ${ }^{1}$ Max-Planck-Institut für Extraterrestrische Physik, Giessenbachstrasse, D-85748 Garching, Germany \\ ${ }^{2}$ Dipartimento di Matematica e Fisica, Università Roma Tre, Via della Vasca Navale 84, I-00146, Roma, Italy \\ ${ }^{3}$ Instituto de Astrofísica de Canarias, E-38205 La Laguna, Tenerife, Spain \\ ${ }^{4}$ Departamento de astrofísica, Univ. de La Laguna, E-38206 La Laguna, Tenerife, Spain \\ ${ }^{5}$ Columbia Astrophysics Laboratory, Columbia University, New York, NY 10027, USA \\ ${ }^{6}$ California Institute of Technology, 1200, E. California Blvd., Pasadena, CA 91125, USA \\ ${ }^{7}$ Nicolaus Copernicus Astronomical Center, Polish Academy of Sciences, Bartycka 18, PL-00-716 Warsaw, Poland \\ ${ }^{8}$ Space Sciences Laboratory, University of California, Berkeley, CA 94720, USA \\ ${ }^{9}$ Cahill Centre for Astronomy and Astrophysics, California Institute of Technology, Pasadena, CA 91125, USA \\ ${ }^{10}$ European Southern Observatory, K. Schwarzschild-Str 2, D-85748 Garching bei Mnchen, Germany \\ ${ }^{11}$ Department of Astronomy, Harvard University, 60 Garden street, Cambridge, MA 02138, USA \\ ${ }^{12}$ National Centre for Radio Astrophysics (TIFR), Post Box 3, Ganeshkhind P.O., Pune 411 007, India \\ ${ }^{13}$ Jet Propulsion Laboratory, California Institute of Technology, Pasadena, CA 91109, USA
}

Accepted 2017 September 18. Received 2017 September 18; in original form 2017 May 13

\begin{abstract}
AX J1745.6-2901 is a high-inclination (eclipsing) transient neutron star (NS) low-mass $\mathrm{X}$-ray binary showcasing intense ionized $\mathrm{Fe} \mathrm{K}$ absorption. We present here the analysis of 11 XMM-Newton and 15 NUSTAR new data sets (obtained between 2013 and 2016), therefore tripling the number of observations of AX J1745.6-2901 in outburst. Thanks to simultaneous $X M M-N e w t o n$ and NUSTAR spectra, we greatly improve on the fitting of the X-ray continuum. During the soft state, the emission can be described by a disc blackbody $(k T \sim 1.1-1.2 \mathrm{keV}$ and inner disc radius $\left.r_{\mathrm{DBB}} \sim 14 \mathrm{~km}\right)$, plus hot $(k T \sim 2.2-3.0 \mathrm{keV})$ blackbody radiation with a small emitting radius $\left(r_{\mathrm{BB}} \sim 0.5-0.8 \mathrm{~km}\right)$ likely associated with the boundary layer or NS surface, plus a faint Comptonization component. Imprinted on the spectra are clear absorption features created by both neutral and ionized matter. Additionally, positive residuals suggestive of an emission $\mathrm{Fe} \mathrm{K} \alpha$ disc line and consistent with relativistic ionized reflection are present during the soft state, while such residuals are not significant during the hard state. The hard-state spectra are characterized by a hard $(\Gamma \sim 1.9-2.1)$ power law, showing no evidence for a high energy cut-off $\left(k T_{\mathrm{e}}>60-140 \mathrm{keV}\right)$ and implying a small optical depth $(\tau<1.6)$. The new observations confirm the previously witnessed trend of exhibiting strong $\mathrm{Fe} \mathrm{K}$ absorption in the soft state that significantly weakens during the hard state. Optical (GROND) and radio (GMRT) observations suggest for AX J1745.6-2901 a standard broad-band spectral energy distribution as typically observed in accreting NSs.
\end{abstract}

Key words: accretion, accretion discs - methods: observational - techniques: spectroscopic stars: neutron - absorption lines - X-rays: binaries.

\section{INTRODUCTION}

Accreting X-ray binaries are amongst the brightest objects of the X-ray sky (Voges et al. 1999). The source population is divided into

*E-mail: ponti@iasfbo.inaf.it several sub-classes. Depending on the nature of the compact object, they are classified as either black holes (BHs) or neutron stars (NSs) X-ray binaries (Liu, van Paradijs \& van den Heuvel 2000, 2001; Tetarenko et al. 2016; Corral-Santana et al. 2016). According to the type of the companion star, they are further separated into either high-mass X-ray binary or low-mass X-ray binary (LMXB). The family of NS LMXB is additionally split into Atoll and 
Z-sources, specified by the shape of their colour-colour X-ray diagram (Hasinger \& van der Klis 1989). The former covers a lower and larger luminosity range ( $\left.L \sim 0.001-0.5 L_{\text {Edd }}\right)$ and they typically show both a soft state and a hard state (Muñoz-Darias et al. 2014). In contrast, Z-sources radiate at luminosities close to Eddington and their spectra are usually soft showing a more complex phenomenology (Barret \& Olive 2002; Reig, van Straaten \& van der Klis 2004; van der Klis 2006).

Despite the differences (such as a surface and its associated emission that is present in NS while it is absent in $\mathrm{BH}$, consequently this induces a softer and lower temperature Comptonized component; Burke, Gilfanov \& Sunyaev 2017), all LMXBs are linked through common features. It was recently shown that NS LMXB displays spectral states and a hysteresis pattern similar to that observed in BH binaries, indicating a physical link between these different sources (Muñoz-Darias et al. 2014). Similarly, both classes of sources display alike fast variability properties (Wijnands \& van der Klis 1999; Belloni, Psaltis \& van der Klis 2002; Belloni, Motta \& Muñoz-Darias 2011; Fender \& Muñoz-Darias 2016; Motta et al. 2017). Regardless of these common behaviours, more complex spectral models have been typically applied to NS LMXB, compared to BH systems (see Barret 2001 for a review). Indeed, the bulk of the emission from $\mathrm{BH}$ systems can generally be reproduced by a soft disc blackbody plus a hard Comptonization component (Done, Gierliński \& Kubota 2007; Dunn et al. 2010, 2011; Plant et al. 2014). While a larger array of models and different approaches have been employed to explain the emission from NS LMXB, partly disguising the similarities (Mitsuda et al. 1984, 1989; White, Stella \& Parmar 1988; Church \& Balucińska-Church 1995, 2001, 2004; Barret et al. 2000; Gierliński \& Done 2002; Iaria et al. 2005).

Mainly based on the lessons learned from the studies of BH systems, Lin, Remillard \& Homan $(2007,2009)$ proposed a new model for NS composed of three main emission components. This model is composed by the same two components required to fit the spectra of $\mathrm{BH}$ binaries (disc blackbody plus Comptonization), with the addition of a blackbody component to reproduce the radiation from the NS boundary layer. It was successfully applied to observations of several Atoll and Z-sources (Lin et al. 2007, 2009; Armas Padilla et al. 2017). In particular, the model allows fitting both the soft- and hard-state observations, obtaining best-fitting parameters similar to those observed in BH systems (besides the boundary layer).

During the soft state, the emission is usually dominated by the thermal components, with characteristic temperatures of $k T \sim 0.5-$ $2.0 \mathrm{keV}$ (Barret et al. 2000; Di Salvo et al. 2000; Oosterbroek et al. 2001; Iaria et al. 2005; Lin et al. 2007, 2009; Armas Padilla et al. 2017). The Comptonized component is weak with low temperature $\left(k T_{\mathrm{e}} \sim\right.$ few tens $\left.\mathrm{keV}\right)$ and large optical depth $(\tau \sim 5-15$, for a spherical geometry). In the hard state, the spectra are dominated by a hard Comptonized component with temperatures of a few tens of $\mathrm{keV}$ and optical depths $(\tau \sim 2-3)$. The thermal components are observed at low temperatures $(k T<1 \mathrm{keV})$ and with significantly lower luminosity.

Thanks to the combination of increased effective area and energy resolution, a number of additional narrow and broad features were detected in the recent years. Indeed, broad $\mathrm{Fe} \mathrm{K} \alpha$ emission lines are often measured (Miller et al. 2006c, 2007; Done \& Gierliński 2006; Reis et al. 2008; Yamada et al. 2009; Tomsick et al. 2009; Done \& Díaz Trigo 2010; Reis, Fabian \& Miller 2010; Shidatsu et al. 2011; Kolehmainen, Done \& Díaz Trigo 2014; Petrucci et al. 2014 ). The shape of such lines carries information about the geometry and the extension of the accretion disc as well as of the extent and location of the primary source (Fabian et al. 1989; Reynolds \& Nowak 2003; Fabian \& Ross 2010).

Additionally, the past decade has seen a burst of detections of ionized absorption lines and edges in high inclination LMXB (Brandt \& Schulz 2002; Lee et al. 2002; Parmar et al. 2002; Ueda et al. 2004; Díaz Trigo et al. 2006; Miller et al. 2006a,b). It is now recognized that winds/ionized absorptions are an ubiquitous component of accreting LMXB during the soft state (Ponti et al. 2012; Ponti et al. 2016a).

The unprecedented focusing capability of the mirrors aboard the NUSTAR telescope provides an improvement of the sensitivity in the $10-79 \mathrm{keV}$ energy range by more than two orders of magnitudes compared to previous coded mask telescopes (Harrison et al. 2013). This opens a new window for the study of both faint $\mathrm{X}$-ray sources, such as the less luminous Atoll sources, as well as sources in crowded regions, such as the Galactic centre (GC; Ponti et al. 2015c).

Looking into the NUSTAR and XMM-Newton archives, we noted that AX J1745.6-2901 is one of the transient LMXB with the best $N u S T A R+X M M-N e w t o n$ publicly available monitoring campaign.

\subsection{AX J1745.6-2901}

AX J1745.6-2901 is a high inclination (dipping and eclipsing) accreting NS transient, classified as an Atoll source and showing clear evidence for highly ionized absorption as well as type I bursts (Maeda et al. 1996; Hyodo et al. 2009; Ponti et al. 2015a). AX J1745.6-2901 is located towards the GC region at a projected distance of less than 1.5 arcmin from Sgr A ${ }^{\star}$ (Degenaar \& Wijnands 2009; Ponti et al. 2015b). The high column density of neutral material $\left(N_{\mathrm{H}} \sim 3 \times 10^{23} \mathrm{~cm}^{-2}\right)$ as well as the spatial distribution of the dust scattering halo suggests that AX J1745.6-2901 is either at or behind the GC, therefore at a distance $\geq 8 \mathrm{kpc}$ (Jin et al. 2017; Ponti et al. 2017 ). The source has an orbital period of $8.35100811 \pm 0.00000002 \mathrm{~h}$ decreasing with time at a rate of $\dot{P}_{\text {orb }}=-4.03 \pm 0.32 \times 10^{-11} \mathrm{~s} \mathrm{~s}^{-1}$, suggesting non-conservative mass transfer (Maeda et al. 1996; Ponti et al. 2016b).

Being located at less than 1.5 arcmin from Sgr A*, AX J1745.62901 is one of the best monitored accreting compact objects, having deep and frequent XMM-Newton and NuSTAR observations (see Ponti et al. 2015b,c). AX J1745.6-2901 was discovered by ASCA during the 1993-1994 outburst (Maeda et al. 1996). The source recently showed strong activity with two short ( $\sim 4$ months) outbursts in 2006 and 2007, as well as two long and luminous outbursts in 2007-2008 and 2013-2016 (Degenaar et al. 2013; Ponti et al. 2015a). The latest ended in June 2016 (Degenaar et al. 2016). The intense activity together with the frequent X-ray monitoring of this field make AX J1745.6-2901 one of the best candidates to investigate the evolution of the X-ray emission and constrain the spectral energy distribution (SED) in this low luminosity GC Atoll source. This will be instrumental to investigate, in a following paper (Bianchi et al. 2017), whether the ionized absorption observed towards AX J1745.6-2901 dissolves in the hard state because it becomes unstable for photoionization.

We report here 11 new XMM-Newton observations (4 and 7 of which are in the soft and hard states, respectively) as well as 15 new NUSTAR observations that caught AX J1745.6-2901 in outburst. In particular, we report the results of a set of five strictly simultaneous $X M M$-Newton $+N u S T A R$ observations, excellent for characterizing the X-ray SED above $10 \mathrm{keV}$ within the crowded GC region (Ponti et al. 2015b). 
Table 1. Best-fitting parameters of the new XMM-Newton soft- and hard-state spectra, once fitted with single components absorbed models. The soft-state spectra are best fit with absorbed thermal models with the additional absorption by ionized material, while the hard-state spectra are best fit by absorbed power-law emission. The best-fitting emissivity profile of the disc line is $r^{-2.4 \pm 0.1}$ reflected off a disc with an inclination of $i=70_{-15}^{+7}$. During the soft state, the neutral and ionized absorption are consistent with being constant over time. However, the ionized absorption component disappears during the hard state. This is consistent with the results from archival observations. $\dagger$ in units of $10^{-4}$ photons cm $\mathrm{cm}^{-2}$; $\ddagger$ in units of $10^{-2}$ ph $\mathrm{keV}^{-1} \mathrm{~cm}^{-2} \mathrm{~s}^{-1}$ at $1 \mathrm{keV}$; b normalization $N_{\mathrm{DBB}}=R_{\mathrm{DBB}}^{2} / D_{10}^{2} \cos (\theta)$, where $R_{\mathrm{DBB}}$ is the apparent inner disc radius in km, $D_{10}$ is the distance to the source in units of $10 \mathrm{kpc}$ and $\theta$ the angle of the disc $(\theta=0$ is face on).

\begin{tabular}{|c|c|c|c|c|c|c|c|}
\hline \multicolumn{8}{|c|}{ tbabs*axjdust*IA*(diskbb+diskline $)$} \\
\hline OBSID & $\begin{array}{c}N_{\mathrm{H}} \\
\left(10^{22} \mathrm{~cm}^{-2}\right)\end{array}$ & $\log \left(\xi_{I A}\right)$ & $\log \left(N_{\mathrm{H}_{\mathrm{IA}}}\right)$ & $\begin{array}{c}N_{\mathrm{dl}} \\
\dagger\end{array}$ & $\begin{array}{c}k T \\
(\mathrm{keV})\end{array}$ & $\begin{array}{c}N_{\mathrm{DBB}} \\
b\end{array}$ & $\chi^{2} /$ dof \\
\hline \multicolumn{8}{|c|}{$X M M$-Newton soft state } \\
\hline 0743630901 & $29.2 \pm 1.1$ & $4.0_{-0.1}^{+0.7}$ & $23.2_{-0.4}^{+0.6}$ & $12 \pm 5$ & $2.37 \pm 0.10$ & $1.1 \pm 0.2$ & $642.8 / 676$ \\
\hline 0743630801 & $28.8 \pm 0.6$ & $3.9 \pm 0.2$ & $23.4 \pm 0.4$ & $10 \pm 4$ & $2.34 \pm 0.06$ & $1.1 \pm 0.1$ & $647.9 / 676$ \\
\hline \multirow[t]{2}{*}{0743630601} & $29.1 \pm 0.6$ & $4.0 \pm 0.2$ & $23.4_{-0.2}^{+0.3}$ & $6 \pm 2$ & $2.14 \pm 0.05$ & $1.5 \pm 0.1$ & $630.0 / 676$ \\
\hline & $29.6 \pm 0.4$ & $4.4 \pm 0.1$ & $23.9 \pm 0.3$ & & & & \\
\hline \multicolumn{8}{|c|}{ tbabs*axjdust*power-law } \\
\hline OBSID & $\begin{array}{c}N_{\mathrm{H}} \\
\left(10^{22} \mathrm{~cm}^{-2}\right)\end{array}$ & & & & $\Gamma$ & $\begin{array}{c}N_{\mathrm{pl}} \\
\ddagger\end{array}$ & $\chi^{2} /$ dof \\
\hline \multicolumn{8}{|c|}{ XMM-Newton hard state } \\
\hline 0743630501 & $28.9 \pm 1.1$ & & & & $1.98 \pm 0.07$ & $1.8 \pm 0.3$ & $609.9 / 578$ \\
\hline 0743630401 & $29.6 \pm 1.5$ & & & & $2.06 \pm 0.09$ & $2.1 \pm 0.4$ & $624.2 / 578$ \\
\hline 0743630301 & $27.1 \pm 1.4$ & & & & $1.91 \pm 0.09$ & $1.5 \pm 0.3$ & $596.4 / 578$ \\
\hline 0743630201 & $29.3 \pm 1.3$ & & & & $2.12 \pm 0.08$ & $2.4 \pm 0.4$ & $545.0 / 578$ \\
\hline 0723410501 & $29.8 \pm 1.3$ & & & & $1.95 \pm 0.08$ & $1.3 \pm 0.2$ & $576.7 / 578$ \\
\hline 0723410401 & $27.9 \pm 1.3$ & & & & $1.97 \pm 0.08$ & $1.3 \pm 0.2$ & $595.3 / 578$ \\
\hline 0723410301 & $29.4 \pm 1.0$ & & & & $1.98 \pm 0.06$ & $2.3 \pm 0.3$ & $614.4 / 578$ \\
\hline
\end{tabular}

\section{OBSERVATIONS AND DATA REDUCTION}

All spectral fits were performed using the XSPEC software package (version 12.7.0; Arnaud 1996). Uncertainties and upper limits are reported at the 90 per cent confidence level for one interesting parameter, unless otherwise stated. The reported X-ray fluxes are not corrected for Galactic absorption. All luminosities, blackbody and disc blackbody radii assume that AX J1745.6-2901 is located at the GC distance, therefore $d_{\mathrm{AXJ}}=8.3 \mathrm{kpc}$ (Genzel et al. 2010; Bland-Hawthorn \& Gerhard 2016; Gillessen et al. 2016). To derive the disc blackbody inner radius $r_{\mathrm{DBB}}$, we fit the spectrum with the disc blackbody model (Mitsuda et al. 1984; Makishima et al. 1986) the normalization of which provides the apparent inner disc radius $\left(R_{\mathrm{DBB}}\right)$. Following Kubota et al. (1998), we correct the apparent inner disc radius through the equation: $r_{\mathrm{DBB}}=\xi \kappa^{2} R_{\mathrm{DBB}}$ (where $\kappa=2$ and $\left.\xi=\sqrt{(3 / 7)} \times(6 / 7)^{3}\right)$ in order to estimate the likely inner disc radius $r_{\mathrm{DBB}}$. We also assume an inclination of the accretion disc of $80^{\circ}$ (AX J1745.6-2901 is an eclipsing source). We adopt a nominal Eddington limit for AX J1745.6-2901 of $\mathrm{L}_{\mathrm{Edd}}=2 \times 10^{38} \mathrm{erg}$ $\mathrm{s}^{-1}$ (appropriate for a primary mass of $M_{\mathrm{NS}} \sim 1.4 \mathrm{M}_{\odot}$ and cosmic composition; Lewin, van Paradijs \& Taam 1993). To allow the use of $\chi^{2}$ statistics we group each spectrum to have a minimum of 30 counts in each bin. We fit the interstellar absorption with either the TBABS or the TBVARABS models in XSPEC, assuming Wilms, Allen \& McCray (2000) abundances and Verner et al. (1996) cross-sections.

\subsection{XMM-Newton}

We followed the same data reduction steps (e.g. filtering out bursts and dipping periods) employed by Ponti et al. (2015a). We reanalysed all the data sets considered in that work, with the latest version (15.0.0) of the XMM-Newton (Jansen et al. 2001; Strüder et al. 2001; Turner et al. 2001) Science Analysis System SAS, applying the most recent (as of 2016 September 2) calibrations (see Table 1 of Ponti et al. 2015a). We also report the analysis of 11 new XMM-Newton observations of AX J1745.6-2901 in outburst (the study of other sources within the field of view of the same observations are included in Ponti et al. 2015b,c, 2016a,b). Table A1 shows the details of all the new XMM-Newton observations considered in this work (see table 1 of Ponti et al. 2015a for the old observations). All the new observations have been accumulated in Full Frame mode with the medium filter applied. Pile-up distorts the source spectra in the soft state. We therefore adopt an annular extraction region, while a circular one is considered in the hard state (see Ponti et al. 2015a). We corrected the spectra of AX J1745.6-2901 for the effects of the dust scattering halo (see Jin et al. 2017). During obsid 0790180401 the spectrum of AX J1745.6-2901 is affected by the emission from Swift J174540.7-290015, characterized by a very soft spectrum (see Ponti et al. 2016b). To avoid possible biases, we decided to disregard this data set from further analysis.

\subsection{NUSTAR}

We analysed all NuSTAR (Harrison et al. 2013) public (as of 2016 September 2) observations that caught AX J1745.6-2901 in outburst, resulting in 15 separate data sets (see Table A2). We reduced and cleaned the data with the latest version of the NUSTAR CALDB (20161021). We used the NuSTAR Data Analysis Software (NUSTARDAS) sub-package v1.6.0 that is part of the HEASOFT v.6.19 (see Wik et al. 2014; Madsen et al. 2015 for more information about the calibration of the NUSTAR observatory). The NUSTAR data were reduced with the standard nupipeline scripts v. 0.4.5 (released on 2016 March 30) and the high-level products produced with the nuproducts tool. The source photons were extracted from a circular region of 70 arcsec radius, centred on the source (Fig. A1). 
Response matrices appropriate for each data set were generated using the standard software. We did not combine modules.

Bursts have been removed by generating a light curve with $3 \mathrm{~s}$ time binning in the $5-10 \mathrm{keV}$ energy band and cutting all intervals with a count rate higher than the threshold reported in Table A2. We also filtered out eclipses and dips by producing a light curve in the same energy band (with $60 \mathrm{~s}$ and $180 \mathrm{~s}$ time bins for the softand hard-state observations, respectively) and dismissing all periods with count rate lower than the threshold reported in Table A2.

In Appendix A (Section A), we report more details on the treatment of the bright point sources contaminating the spectrum of AX J1745.6-2901 (Section A1), on the treatment of the background emission (Section A2), as well as the potential mismatch at low energy between the spectrum measured by NUSTAR and by XMMNewton (Section A3). As detailed in Section A3, we limit the $N u S$ TAR spectral analysis to the 5.5-40 and 3-70 keV energy range during the soft- and hard-state, respectively.

\subsection{Swift}

Following the same procedure described in Ponti et al. (2015a), we have extracted a Swift-XRT light curve of AX J1745.6-2901 from all 'photon counting' mode observations available as of 2016 September 2.

\subsection{GROND}

On 2015 April 29, AX J1745.6-2901 was observed during the soft X-ray spectral state with the Gamma-Ray burst Optical Nearinfrared Detector GROND (Greiner et al. 2008) at the MPG $2.2 \mathrm{~m}$ telescope in La Silla, Chile. GROND provides simultaneous imaging in four optical $\left(g^{\prime}, r^{\prime}, i^{\prime}, z^{\prime}\right)$ and three near-infrared $\left(J, H, K_{s}\right)$ channels. Owing to the extreme Galactic foreground reddening towards the target $(E(B-V) \sim 86 \mathrm{mag}$; Schlafly \& Finkbeiner 2011), only the analysis of the near-IR bands is discussed here.

The data were reduced with the standard tools and methods described in Krühler et al. (2008). To search for a near-IR counterpart to AX J1745.6-2901, we combined the best seeing images taken during the night, providing total integration times of 94,72 and $96 \mathrm{~min}$ in $J, H$ and $K_{s}$, respectively. The resulting medians full width at halfmaximum (FWHM) of the point spread function (PSF) were 1.1, 1.6 and 1.2 arcsec in the three bands, respectively. The astrometric solution, with an accuracy of 0.01 arcsec in both coordinates, was obtained using selected 2-Micron All-Sky Survey (2MASS) field stars (Skrutskie et al. 2006). The photometry was measured from apertures with sizes corresponding to the image FWHM and calibrated against 2MASS field stars. This resulted in $1 \sigma$ systematic uncertainties of $0.10(J), 0.09(H)$ and $0.17 \mathrm{mag}\left(K_{s}\right)$.

Fig. 1 (left) shows the $J$-band image of the region around AX J1745.6-2901 indicating the extreme crowding characteristic of the GC region. Fig. 1 (right) provides a zoom-in of the $K_{s}$-band image. We detect emission consistent with the $3 \sigma$ uncertainty of the Chandra position (see Jin et al. 2017), but the available angular resolution does not allow us to distinguish between either emission from a single source or from the superposition of multiple objects. Therefore, we consider the photometry measurements of $J>19$, $H>15.5$ and $K_{s}>14.5$ (all in the Vega system), obtained with an aperture centred at the Chandra position, as upper limits on the observed magnitude of any counterpart to AX J1745.6-2901. These magnitude upper limits correspond to flux densities of $J<4 \times 10^{-5}$, $H<7 \times 10^{-4}$ and $K<1 \times 10^{-3} \mathrm{Jy}$.

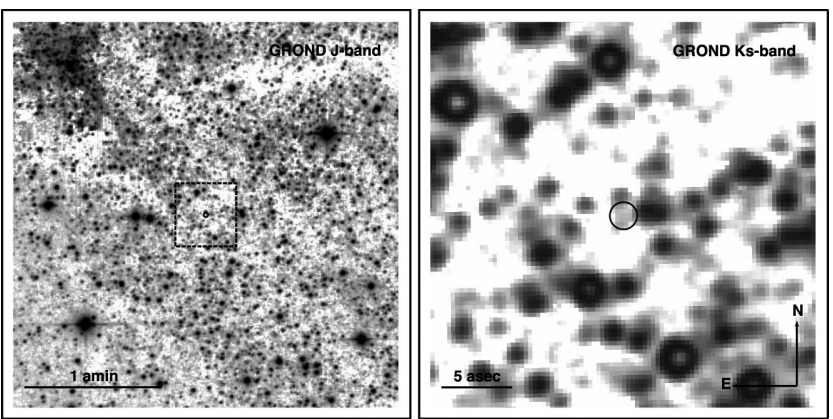

Figure 1. Left: GROND J-band image of the field of AX J1745.6-2901. The $3 \sigma$ Chandra location uncertainty with radius of 0.96 arcsec is indicated by the black circle (Jin et al. 2017). The central star cluster surrounding Sgr $\mathrm{A}^{\star}$ appears in the top left corner of the image. Right: Zoom-in into the $K_{s}$-band image of the region shown by the dashed rectangle in the left image.

Constraints on the donor star can be derived with an assumption on the distance to the source $(\approx 8.3 \mathrm{kpc})$ and a good approximation of the Galactic foreground reddening. Using $A_{J} \approx 7.4 \mathrm{mag}, A_{H}$ $\approx 4.2 \mathrm{mag}$ and $A_{K} \approx 2.4 \mathrm{mag}$ (Fritz et al. 2011), the limits on the absolute magnitude of the companion star are estimated to be $M_{J}>-3.0 \mathrm{mag}, M_{H}>-3.3 \mathrm{mag}$ and $M_{\mathrm{K}}>-2.5 \mathrm{mag}$. This is in agreement with the classification of AX J1745.6-2901 as an LMXB and suggests the donor to belong to the stellar luminosity class III (giants) or fainter.

\subsection{GMRT}

The Giant Metrewave Radio Telescope (GMRT) is a multielement aperture synthesis telescope consisting of 30 antennas, each with a diameter of $45 \mathrm{~m}$, separated by a maximum baseline of $25 \mathrm{~km}$ (Swarup et al. 1997). We observed the GC region (centred at Sgr $\mathrm{A}^{\star}$ ) at $1390 \mathrm{MHz}$ on 2015 August 13.6 (UT) for $8 \mathrm{~h}$ (DDTB178). Thanks to a simultaneous Chandra HETG observation, we can establish that AX J1745.6-2901 was in the soft state during the GMRT monitoring. The primary beam FWHM of the GMRT is 24 arcmin at $1.4 \mathrm{GHz}$, and hence, AX J1745.6-2901 was well within the field of view of the observation. A total of 33.33 $\mathrm{MHz}$ bandwidth divided into 256 frequency channels was used with an integration time of $16.1 \mathrm{~s}$. 3C 48 was used as the flux density calibrator, while the sources $1822-096$ and 1751-253 were used as phase calibrators.

The data were analysed using the Astronomical Image Processing System. After flagging the original data set for non-working antennas and radio frequency interference, data from a single frequency channel of the flux and phase calibrators were used to construct timebased amplitude and phase calibrations, while bandpass calibration was done with the flux calibrator. The bandwidth was divided into 32 channels of $1 \mathrm{MHz}$ each before imaging in order to minimize the effects of bandwidth smearing. A high-resolution map of the region (imaged with baselines $1.5 k \lambda<b<120 k \lambda$ ) was first constructed to resolve the diffuse emission in the region, followed by self-calibration. The clean components from this image (containing the resolved diffuse emission) were then subtracted from the original $(u, v)$ data, which was used to construct a low-resolution map of the region (baselines $0 k \lambda<b<5 k \lambda$ ). In order to remove the confusing diffuse emission from the original map, the clean components from this low-resolution image were then subtracted from the original $(u, v)$ data. The final $(u, v)$ data set was then used to 


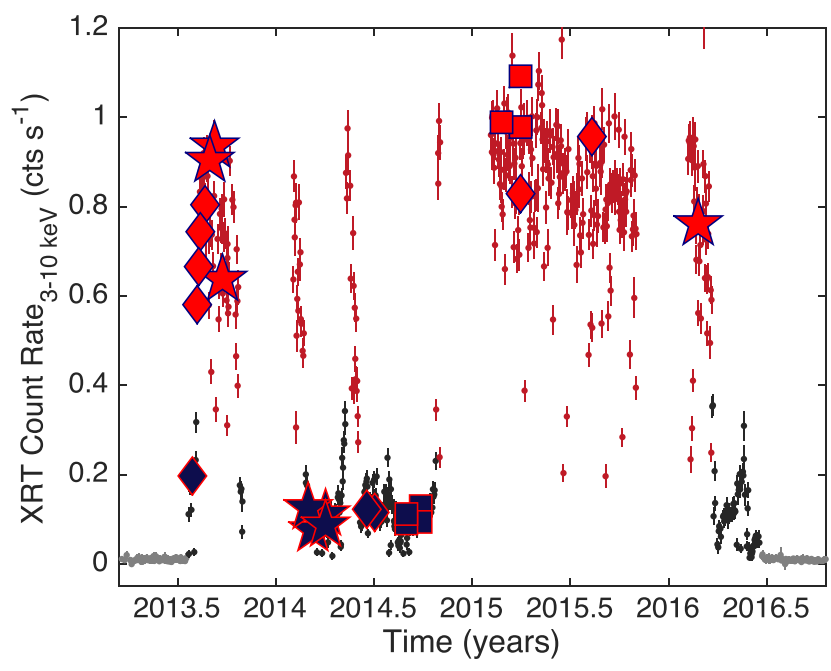

Figure 2. Swift-XRT light curve of the latest outburst of AX J1745.62901. The red, black and grey points indicate the soft, hard and quiescent source states, respectively (see text for more details). The stars, squares and diamonds indicate the time and equivalent Swift-XRT count rate for each of the XMM-Newton, NUSTAR and simultaneous (XMM-Newton+NuSTAR) observations, respectively. Red and black symbols manifest observations that caught AX J1745.6-2901 in the soft and hard states, respectively. We do not remove Swift exposures (partially) accumulated during eclipses.

construct a high-resolution map (baselines $3 k \lambda<b<120 k \lambda$ ) of the region with $3 \mathrm{D}$ imaging over 31 facets, which was subsequently self-calibrated.

The high crowding and diffuse emission around the region limited the map rms to $\approx 0.8 \mathrm{mJy}_{\text {beam }}{ }^{-1}$. No radio source was identified at the position of AX J1745.6-2901 with a $3 \sigma$ upper limit of $2.4 \mathrm{mJy}$. The corresponding radio luminosity upper limit for the source is $2.7 \times 10^{29} \mathrm{erg} \mathrm{s}^{-1}$ at $1.4 \mathrm{GHz}$.

\section{ACCRETION STATES AND X-RAY LIGHT CURVE}

The dots in Fig. 2 display the evolution of the 3-10 keV emission of AX J1745.6-2901 during its last outburst, as observed by Swift-XRT (see Degenaar et al. 2014 and Ponti et al. 2015a for an historical light curve). The colour code of the circles provides an estimate of the source state ${ }^{1}$ (e.g. grey, black and red points correspond to the quiescence, hard and soft states, respectively). Although the determination of the spectral state based uniquely on Swift data is fairly uncertain (because of the large uncertainties associated with the X-ray colour within the short Swift-XRT exposures) we note that they, in all cases, agree with the states deduced from the higher quality XMM-Newton and NuSTAR data (Figs 2 and 3).

The light curve of AX J1745.6-2901 exhibits many interesting behaviours. During the latest outburst, AX J1745.6-2901 went at least four times through the entire hysteresis loop (from a hard to

\footnotetext{
${ }^{1}$ We determine the state of AX J1745.6-2901 based on X-ray colours. However, we point out that the presence of eclipses and dips, which significantly modify the observed X-ray colours, make the determination of the source state very challenging within the short (typically $0.5-1 \mathrm{ks}$ ) Swift exposures. We tentatively associate with a soft state the Swift observations with an average 3-10 keV flux in excess of $5 \times 10^{-10} \mathrm{erg} \mathrm{cm}^{-2} \mathrm{~s}^{-1}$ and hardness lower than 1.4 (e.g. see Fig. 3). However, to reduce the effect of dips and eclipses, the average is computed over five consecutive Swift observations.
}

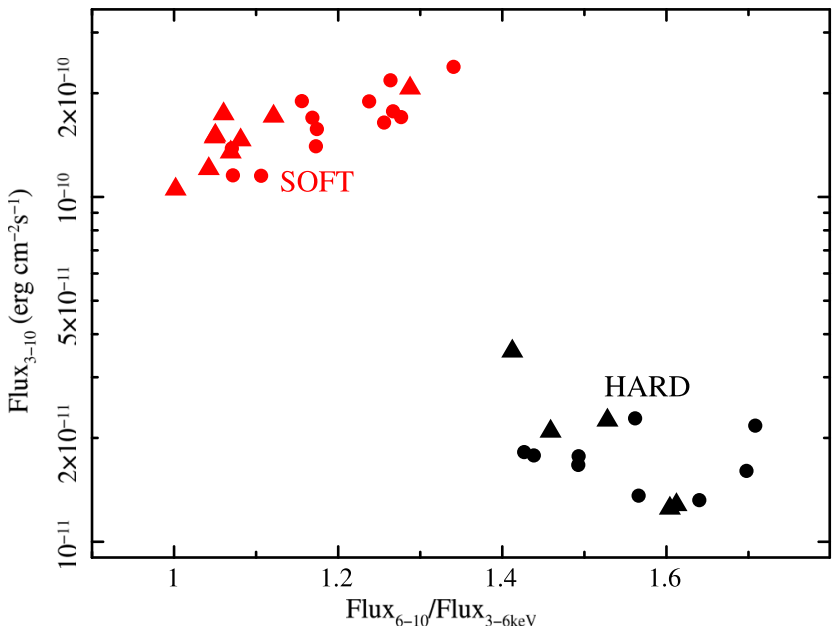

Figure 3. Hardness intensity diagram (HID) of AX J1745.6-2901 derived from each XMM-Newton and NUSTAR observation (they are represented with circles and triangles, respectively). Red and black symbols indicate soft and hard states, respectively. The two states are clearly separated.

soft and back to the hard state), before going back to quiescence. This behaviour is observed in several other NS LMXB and it appears starkly different from outbursts of BH LMXB, which typically perform the hysteresis loop only once (Muñoz-Darias et al. 2014; Ponti, Muñoz-Darias \& Fender 2014). Similar to Ponti et al. (2015a), we determine the state of the source on the basis of the X-ray colour. Fig. 3 shows the hardness intensity diagram (HID), which is often used to determine the source state (Fender, Belloni \& Gallo 2004; Belloni et al. 2011; Muñoz-Darias et al. 2014). The hardness is defined here as the ratio between the observed fluxes in the 6-10 and 3-6 keV bands. As it is rather widespread in Atoll sources, we note that during all XMM-Newton and NuSTAR observations (which allow us to securely pin down the state of the source) the flux of AX J1745.6-2901 during the hard state is in all cases significantly lower than in the soft state (see Figs 2 and 3).

\section{NEW XMM-NEWTON OBSERVATIONS}

\subsection{XMM-Newton soft state}

The top panel of Fig. 4 shows the combined spectrum of the three new XMM-Newton soft-state observations (Table A1). We simultaneously fit the three spectra with the same model, however allowing the parameters to be different between the three spectra as detailed below, and plot the combined data and residuals. Consistent with what was observed in archival observations (Ponti et al. 2015a), the $\mathrm{X}$-ray emission is absorbed by a large column density of neutral (lowly ionized) equivalent hydrogen also during the new XMMNewton observations (Fig. 4).

We start by simultaneously fitting these three new soft-state $X M M$-Newton spectra with a disc blackbody model, absorbed by neutral material and modified by dust scattering effects (TBABS*AXJDUST*DISKBB in XSPEC; see Jin et al. 2017 for a full description of the AXJDUST component). The free parameters in the fit of each spectrum are the column density of neutral hydrogen absorption $\left(N_{\mathrm{H}}\right)$, the disc blackbody temperature $\left(k T_{\mathrm{DBB}}\right)$ and disc normalization $\left(N_{\mathrm{DBB}}\right)$. The fit leaves highly significant residuals between $\sim 6$ and $8 \mathrm{keV}$, clear signatures of the presence of an additional 


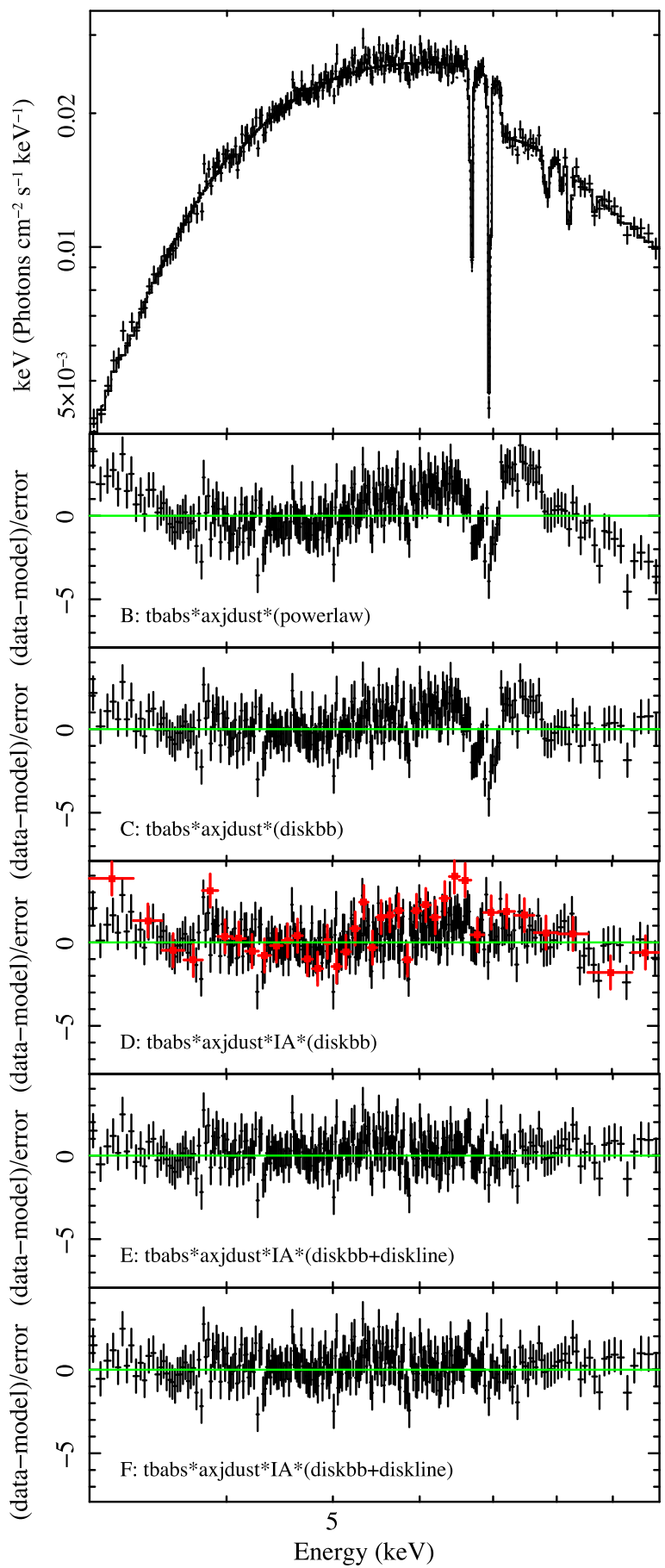

Figure 4. XMM-Newton combined spectrum of the three new soft-state observations (Table A1). Top panel: Unfolded spectrum, fitted with the bestfitting model, composed by disc blackbody plus disc-line emission absorbed by neutral and ionized material (TBABS*AXJDUST*IA*(DISKBB+DISKLINE)). Panel B: Observed residuals, once the spectra are fitted with an absorbed power-law model (TBABS*AXJDUST*(POWERLAw)). Panel C: As panel $\mathrm{B}$, once the power law is substituted with a disc blackbody model (TBABS*AXJDUST*(DISKBв)). Clear residuals are still present. Panel D: As panel $\mathrm{C}$, once an ionized absorption model component is added to the fit (TBABS*AXJDUST*IA*(DISKBB)). The red points show the residuals, once the data are heavily rebinned. Small remaining residuals in the $5-8 \mathrm{keV}$ band are still visible. Panel E: Observed residuals, once a broad $\mathrm{Fe} \mathrm{K} \alpha$ emission line is added to the best fit [TBABS*AXJDUST*IA*(DISKBB+DISKLINE)]. Panel F: As panel E, once forcing the ionized and neutral absorption component to be constant over time, between the different soft-state observations. ionized absorption component (see panel C of Fig. $4 ; \chi_{\mathrm{DBB}}^{2}=$ 2162.5 for 2037 degree of freedom - dof).

Before investigating further the nature of the residuals in the $\sim 6-$ $8 \mathrm{keV}$ band, we tested the shape of the broad-band spectrum. We first substituted the thermal disc blackbody component with a power law (TBABS*AXJDUST*POWERLAW) with $N_{\mathrm{H}}$, power-law photon index $\Gamma$ and normalization $N_{\mathrm{pl}}$ as free parameters. We observed a significantly worse fit compared to the thermal disc blackbody model (see panel B of Fig. $4 ; \chi_{\mathrm{PL}}^{2}=2525.5$ for 2037 dof). Therefore, we can exclude that the continuum during these soft-state spectra can be reproduced by a power law. Finally, we exchanged the disc blackbody component with a blackbody one (TBABS*AXJDUST ${ }^{*}$ BBODY) with $N_{\mathrm{H}}$, blackbody temperature $k T_{\mathrm{BB}}$ and blackbody radius $r_{\mathrm{BB}}$ as free parameters. In this case, the fit is acceptable and comparable to the one employing the disc blackbody continuum $\left(\chi^{2}=2135.8\right.$ for 2037 dof). We concluded that, consistently with the results obtained from archival data (Ponti et al. 2015a), the new soft-state observations are consistent with an absorbed thermal continuum model, while a power law is excluded. We also noted that the two thermal continuum models appear degenerate within the limited XMM-Newton energy band. We, therefore, report in the rest of this section only the results obtained by fitting the spectra with the disc blackbody model. We also note that the inferred best-fitting inner disc radius results to be $r_{\mathrm{DBB}} \sim 3.5 \mathrm{~km}$ smaller than the NS radius. This suggests that the underlying continuum is more complex than statistically required by the XMM-Newton data alone. Indeed, multiple emission components considering either and both blackbody and disc blackbody components will be explored whenever simultaneous NuSTAR data will be available; Tables 2 and 3 ).

To reduce the residuals observed in the $\sim 6-8 \mathrm{keV}$ energy band, we added a self-consistent ionized absorption component (IA), accurate for the soft-state SED of AX J1745.6-2901 (see Ponti et al. 2015 a for details $)^{2}$. We leave as free parameters for each spectrum the plasma ionization parameter $\left(\log \left(\xi_{\mathrm{IA}}\right)\right)$ and column density $\left(\log \left(N_{\mathrm{H}_{\mathrm{IA}}}\right)\right)$. We leave the absorber outflow velocity $\left(v_{\text {out }}\right)$ free to vary; however, we force it to be the same for all spectra. The addition of such component provides a highly significant improvement of the fit (see panel D of Fig. $4 ; \Delta \chi^{2}=163.6$ ) as well as a satisfactory description of the data ( $\chi^{2}=1998.9$ for 2030 dof $)$.

By heavily rebinning the data (see red points in panel D of Fig. 4), we noted some remaining broad residuals in the $\sim 6-7.5 \mathrm{keV}$ band. This excess emission (panel D of Fig. 4) is observed to be placed red-ward of the absorption features and it appears to be reminiscent of P-Cygni profiles observed in some BH systems and due to the combination of emission and absorption from an outflowing plasma (King et al. 2015; Miller et al. 2015; Munoz-Darias et al. 2016, 2017). Therefore, we model this emission component with the addition of a Gaussian line (TBABS*IA*AXJDUST*(DISKBB+GAUSSIAN)). The line energy and width are free to vary, but tied between the three spectra. The addition of the Gaussian line provides a significant improvement of the fit $\left(\Delta \chi^{2}=79.0\right.$ for six more free parameters; $\chi^{2}=1919.9$ for 2024 dof; $F$-test probability $<10^{-6}$ ).

\footnotetext{
${ }^{2}$ The self-consistent ionized absorption component has been created through the photoionization code Cloudy C13.00 (Ferland et al. 2013). The model ingredients are (1) the soft and hard spectral energy distributions as determined in Ponti et al. (2015a); (2) constant electron density $n_{e}=10^{12} \mathrm{~cm}^{-3}$; (3) ionization parameter in the range $\log \left(\xi / 1 \mathrm{erg} \mathrm{cm} \mathrm{s}^{-1}\right)=23: 24.5$; (4) intervening column density in the range $\log \left(N_{\mathrm{H}} / 1 \mathrm{~cm}^{-2}\right)=23.0: 24.5 ;$; 5 ) turbulent velocity $v_{\text {turb }}=500: 1000 \mathrm{~km} \mathrm{~s}^{-1} ;(6)$ chemical abundances as in table 7.1 of Cloudy documentation.
} 
Table 2. Best-fitting model of the longest simultaneous soft state XMM-Newton and NuSTAR observations of AX J1745.6-2901 (2015 February 25 and 26). The different lines in the Table report: the model name, the equivalent hydrogen column density of neutral absorption (in units of $10^{22}$ atoms $\left.\mathrm{cm}^{-2}\right)$, the logarithm of the ionization parameter $\left(\log \left(\xi_{\mathrm{IA}}\right)\right)$ and column density $\left(\log \left(N_{\mathrm{H}_{\mathrm{IA}}}\right)\right)$ of the ionized absorber, the temperature $\left(k T_{\mathrm{DBB}}\right.$, in $\mathrm{keV}$ ) and normalization $\left(N_{\mathrm{DBB}}\right)$ of the disc blackbody and blackbody components ( $k T_{\mathrm{BB}}$ in $\mathrm{keV}$ and $N_{\mathrm{BB}}$ ) (the disc blackbody normalization is $N_{\mathrm{DBB}}=R_{\mathrm{DBB}}^{2} / D_{10}^{2} \cos (\theta)$, where $R_{\mathrm{DBB}}$ is the apparent inner disc radius in $\mathrm{km}, D_{10}$ is the distance to the source in units of $10 \mathrm{kpc}$ and $\theta$ the angle of the disc; the blackbody normalization is: $N_{\mathrm{BB}}=r_{\mathrm{BB}}^{2} / D_{10}^{2}$, where $r_{\mathrm{BB}}$ is the blackbody radius in $\mathrm{km}$ and $D_{10}$ is the distance to the source in units of $10 \mathrm{kpc})$, the fraction of the Comptonized component $\left(f_{\mathrm{sc}}\right)$, the asymptotic photon index of the power law $(\Gamma)$, electron temperature $\left(k T_{\mathrm{e}}\right.$ in $\left.\mathrm{keV}\right)$ and normalization of the Comptonized component $\left(N_{\mathrm{nth}}\right.$ in $10^{-2}$ units), the normalization of the disc-line component $\left(N_{\mathrm{dl}}\right.$ in units of $\left.10^{-4} \mathrm{photons} \mathrm{cm}^{-2} \mathrm{~s}^{-1}\right)$, the normalization of the ionized-relativistic reflection component $\left(N_{\text {ref }}\right.$ in units of $10^{-26}$ photons $\left.\mathrm{cm}^{-2} \mathrm{~s}^{-1}\right)$, the cross-normalization constants $\left(c_{\mathrm{NuA}}\right.$ and $c_{\mathrm{NuB}}$ for NuSTAR module $\mathrm{A}$ and $\mathrm{B}$, respectively) and statistic ( $\chi^{2}$ and dof).

\begin{tabular}{|c|c|c|c|c|c|c|c|c|}
\hline \multirow[b]{2}{*}{ Model } & \multicolumn{7}{|c|}{ Simultaneous $X M M$-Newton + NuSTAR soft state (2015 February 25 and 26) } & \multirow[b]{2}{*}{ DBB-BB-NTH-RF } \\
\hline & DBB-BB-DL & SIMPDBB-DL & DBB-NTH-DL & BB-NTH-DL & DBB-BB-RR & DBB-NTH-RR & BB-NTH-RR & \\
\hline$N_{\mathrm{H}}$ & $30.9 \pm 0.6$ & $30.2 \pm 0.6$ & $31.1 \pm 0.6$ & $28.2 \pm 0.8$ & $31.6 \pm 0.6$ & $31.4 \pm 0.9$ & $27.9 \pm 0.5$ & $32.2 \pm 0.9$ \\
\hline $\log \left(\xi_{I A}\right)$ & $4.0 \pm 0.2$ & $4.2 \pm 0.2$ & $4.0 \pm 0.2$ & $4.0 \pm 0.2$ & $4.1 \pm 0.2$ & $4.1 \pm 0.2$ & $4.2 \pm 0.2$ & $4.1 \pm 0.2$ \\
\hline $\log \left(N_{\mathrm{H}_{\mathrm{IA}}}\right)$ & $23.5 \pm 0.3$ & $23.8 \pm 0.3$ & $23.5 \pm 0.2$ & $23.5 \pm 0.3$ & $23.7 \pm 0.2$ & $23.6 \pm 0.2$ & $23.6 \pm 0.3$ & $23.5 \pm 0.2$ \\
\hline$k T_{\mathrm{DBB}}$ & $1.77 \pm 0.05$ & $1.70 \pm 0.09$ & $1.63 \pm 0.09$ & & $1.55 \pm 0.11$ & $1.1 \pm 0.1$ & & $1.1 \pm 0.1$ \\
\hline$N_{\mathrm{DBB}}$ & $3.0 \pm 0.4$ & $3.3 \pm 0.6$ & $2.8 \pm 0.9$ & & $5.1 \pm 1.7$ & $7.0 \pm 4.2$ & & $17 \pm 6$ \\
\hline$k T_{\mathrm{BB}}$ & $3.0 \pm 0.1$ & & & $1.06 \pm 0.08$ & $3.1 \pm 0.2$ & & $1.01 \pm 0.09$ & $2.3 \pm 0.2$ \\
\hline$N_{\mathrm{BB}}$ & $0.12 \pm 0.03$ & & & $0.015 \pm 0.011$ & $0.08 \pm 0.04$ & & $7 \pm 3$ & $0.5 \pm 0.2$ \\
\hline$f_{\mathrm{sc}}$ & & $0.63 \pm 0.02$ & & & & & & \\
\hline$\Gamma$ & & $4.6 \pm 0.03$ & $2.2 \pm 0.4$ & $2.6 \pm 0.6$ & & $2.1 \pm 0.3$ & $2.1 \pm 0.2$ & $1.1_{-0.2}^{+1.1}$ \\
\hline$k T_{\mathrm{e}}$ & & & $3.6_{-0.4}^{+1.2}$ & $3.8_{-0.6}^{+0.2}$ & & $3.3 \pm 0.3$ & $3.3 \pm 0.3$ & $4.6_{-2.4}^{+10.3}$ \\
\hline$N_{\text {nth }}$ & & & $2.1_{-2.0}^{+3.8}$ & $1.1 \pm 0.7$ & & $3.7 \pm 2.1$ & $0.8 \pm 0.2$ & $0.01 \pm 0.01$ \\
\hline$N_{\mathrm{dl}}$ & $8.3 \pm 1.4$ & $7.4 \pm 1.3$ & $8.7 \pm 1.2$ & $7.9 \pm 1.5$ & & & & \\
\hline$N_{\text {ref }}$ & & & & & $36 \pm 9$ & $55 \pm 11$ & $57 \pm 8$ & $82 \pm 16$ \\
\hline$c_{\mathrm{NuA}}$ & $1.08 \pm 0.02$ & $1.08 \pm 0.02$ & $1.08 \pm 0.01$ & $1.08 \pm 0.01$ & $1.08 \pm 0.01$ & $1.08 \pm 0.01$ & $1.08 \pm 0.01$ & $1.08 \pm 0.01$ \\
\hline$c_{\mathrm{NuB}}$ & $1.12 \pm 0.02$ & $1.12 \pm 0.02$ & $1.12 \pm 0.01$ & $1.12 \pm 0.01$ & $1.12 \pm 0.01$ & $1.12 \pm 0.01$ & $1.12 \pm 0.01$ & $1.12 \pm 0.01$ \\
\hline$\chi^{2} / \mathrm{dof}$ & $1348.5 / 1360$ & 1397.2/1360 & $1346.9 / 1359$ & $1344.1 / 1359$ & $1318.3 / 1360$ & $1306.9 />1359$ & $1328.2 / 1359$ & $1288.5 / 1357$ \\
\hline
\end{tabular}

Table 3. Best-fitting parameters for the two soft state simultaneous XMM-Newton+NuSTAR observations accumulated on 2015 April 02 and 2015 April 01. See the caption of Table 2 for a description of the parameters.

\begin{tabular}{|c|c|c|c|c|c|c|c|c|}
\hline \multirow[b]{2}{*}{ Model } & \multicolumn{5}{|c|}{$\begin{array}{l}\text { Simultaneous XMM-Newton }+ \text { NuSTAR soft state } \\
\text { (2015 April 02) }\end{array}$} & \multicolumn{3}{|c|}{ (2015 April 01) } \\
\hline & DBB-BB-RR & DBB-NTH-RR & BB-NTH-RR & DBB-BB-NTH-RR & DBB-BB-RR & DBB-NTH-RR & BB-NTH-RR & DBB-BB-NTH-RR \\
\hline$N_{\mathrm{H}}$ & $31.5 \pm 0.6$ & $31.3 \pm 0.9$ & $27.0 \pm 0.6$ & $31.5 \pm 0.9$ & $32.8 \pm 1.0$ & $31.1 \pm 1.3$ & $28.4 \pm 1.2$ & $31.5 \pm 1.2$ \\
\hline $\log \left(\xi_{I A}\right)$ & $4.1 \pm 0.2$ & $4.1 \pm 0.2$ & $4.1 \pm 0.2$ & $4.1 \pm 0.2$ & $3.9 \pm 0.3$ & $3.9 \pm 0.3$ & $4.3 \pm 0.4$ & $3.9 \pm 0.4$ \\
\hline $\log \left(N_{\mathrm{H}_{I A}}\right)$ & $23.6 \pm 0.3$ & $23.7 \pm 0.3$ & $23.6 \pm 0.3$ & $23.6 \pm 0.3$ & $23.3 \pm 0.4$ & $23.3 \pm 0.3$ & $23.6 \pm 0.5$ & $23.2 \pm 0.5$ \\
\hline$k T_{\mathrm{DBB}}$ & $1.3 \pm 0.1$ & $1.2 \pm 0.1$ & - & $1.2 \pm 0.2$ & $1.4 \pm 0.3$ & $1.05 \pm 0.4$ & - & $1.02 \pm 0.15$ \\
\hline$N_{\mathrm{DBB}}$ & $8.8 \pm 2.4$ & $8.8 \pm 2.1$ & - & $12 \pm 3$ & $9 \pm 4$ & $7 \pm 5$ & - & $16 \pm 3$ \\
\hline$k T_{\mathrm{BB}}$ & $2.8 \pm 0.2$ & - & $1.08 \pm 0.07$ & $2.6 \pm 0.2$ & $2.7 \pm 0.3$ & - & $1.0 \pm 0.3$ & $2.2 \pm 0.1$ \\
\hline$N_{\mathrm{BB}}$ & $0.2 \pm 0.1$ & - & $11 \pm 5$ & $0.4 \pm 0.2$ & $0.3 \pm 0.2$ & - & $6 \pm 5$ & $0.9 \pm 0.3$ \\
\hline$\Gamma$ & - & $1.4 \pm 0.4$ & $1.4 \pm 0.5$ & $1.1 \dagger$ & - & $1.7 \pm 0.1$ & $1.7 \pm 0.2$ & $1.1 \dagger$ \\
\hline$k T_{\mathrm{e}}$ & - & $2.9 \pm 0.2$ & $2.8 \pm 0.2$ & $4.5 \dagger$ & - & $2.9 \pm 0.1$ & $2.9 \pm 0.2$ & $3.4 \dagger$ \\
\hline$N_{\text {nth }}$ & - & $0.7_{-0.6}^{+1.3}$ & $0.3_{-0.2}^{+0.4}$ & $0.003 \pm 0.002$ & - & $3.2 \pm 1.3$ & $0.8 \pm 0.3$ & $0.01 \pm 0.01$ \\
\hline$N_{\text {ref }}$ & $76 \pm 14$ & $76 \pm 13$ & $73 \pm 16$ & $85 \pm 15$ & $63 \pm 30$ & $83 \pm 22$ & $75 \pm 15$ & $100 \pm 28$ \\
\hline$c_{\mathrm{NuA}}$ & $1.09 \pm 0.02$ & $1.09 \pm 0.01$ & $1.09 \pm 0.02$ & $1.09 \pm 0.02$ & $1.11 \pm 0.02$ & $1.11 \pm 0.02$ & $1.11 \pm 0.02$ & $1.11 \pm 0.02$ \\
\hline$c_{\mathrm{NuB}}$ & $1.12 \pm 0.02$ & $1.12 \pm 0.01$ & $1.12 \pm 0.02$ & $1.12 \pm 0.02$ & $1.20 \pm 0.02$ & $1.20 \pm 0.02$ & $1.20 \pm 0.02$ & $1.20 \pm 0.02$ \\
\hline$\chi^{2} /$ dof & $1284.8 / 1271$ & $1283.6 / 1270$ & $1304.4 / 1270$ & $1280.6 / 1270$ & $1257.5 / 1306$ & $1245.6 / 1305$ & $1249.8 / 1305$ & $1241.6 / 1305$ \\
\hline
\end{tabular}

The best-fitting energy, width and equivalent width of the line are $E=6.43 \pm 0.25 \mathrm{keV}, \sigma=0.85_{-0.17}^{+0.23} \mathrm{keV}$ and $\mathrm{EW} \sim 140-200 \mathrm{eV}$. The line is too broad to be the redshifted emitted component of the same outflowing plasma producing the absorption features. Indeed, the observed line broadening $(\sigma \sim 0.7-1.0 \mathrm{keV})$ would require bulk outflows of $v_{\text {out }} \sim 0.1 \mathrm{c}$. We can rule out that the ionized absorbing plasma is outflowing at such a large speed (e.g. $v_{\text {out }}<2000 \mathrm{~km} \mathrm{~s}^{-1}$ ).

Instead, such residual emission might be due to a broad emission line, reflected off the accretion disc. Indeed, in such a scenario, the disc line would be expected to appear highly broadened as a consequence of the high disc inclination. We, therefore, added a disc line component to the fit (TBABS*IA*AXJDUST*(DISKBB+DISKLINE)), assuming the line energy, inner and outer disc radii to have the values: $E=6.4 \mathrm{keV}, r_{\text {in }}=6$ and $r_{\text {out }}=10^{3} r_{g}\left(r_{g}=G M / c^{2}\right.$, where $G$ is the gravitational constant, $M$ is the mass of the compact object and $c$ is the speed of light). We also assumed the same emissivity profile and disc inclination, for all spectra. We observed a highly significant improvement of the fit (compared to the same model without the disc line) adding such a disc line component to the model (see panel E of Fig. $4 ; \Delta \chi^{2}=78.1$ for 6 more free parameters; $\chi^{2}=1920.8$ 
for 2024 dof). The best-fitting disc inclination is $i=70_{-15}^{+7}$ (on the lower range expected for eclipsing systems, however consistent with the eclipsing nature of the source) and the emissivity index is scaling with disc radius as $r^{-2.4 \pm 0.1}$. The line is observed to have an equivalent width in the range of $E W=120-200 \mathrm{eV}$, therefore consistent with reflection off a standard accretion disc (Matt, Fabian $\&$ Ross 1993). This fit provides an acceptable description of the data (Table 1 and Fig. 4).

Alternatively, the residuals observed in the Fe $\mathrm{K}$ band might be associated with an improper characterization of the Fe K edge imprinted by the neutral absorption (e.g. due to abundances different from Solar or due to depletion; Ponti et al. 2016a). To test this latter possibility, we fitted the data without a broad iron line component, but leaving the iron abundance of the neutral absorber free to vary. We observed only a marginal improvement of the fit $\left(\Delta \chi^{2}=4.9\right.$ for 1 more dof), with a best-fitting iron abundance of $\mathrm{Fe}=1.13_{-0.09}^{+0.16}$ Solar. Therefore, we do not consider this alternative possibility any further.

We observed that all parameters of both the highly and minimally ionized absorption ${ }^{3}$ components are consistent with being constant amongst the new observations as well as consistent with previous observations (see Table 1 and Ponti et al. 2015a). ${ }^{4}$ We, therefore, fit all the new soft-state spectra forcing the absorption to remain constant over time. As a result the best-fitting parameters are $N_{\mathrm{H}}=29.0 \pm 0.4 \times 10^{22} \mathrm{~cm}^{-2}, \log \left(\xi_{\mathrm{IA}}\right)=3.92 \pm 0.16$, $\log \left(N_{\mathrm{H}_{\mathrm{IA}}}\right)=23.3 \pm 0.3$ and $v_{\text {turb }}=700 \mathrm{~km} \mathrm{~s}^{-1}$ and no significant variation of the fit is observed (see panel F of Fig. $4 ; \chi^{2}=1926.0$ for 2030 dof, $\Delta \chi^{2}=-5.2$ for the elimination of six dof; Table 1).

Hereinafter, we will refer to the combination of absorption and scattering effects TBABS $*_{I A} *_{\text {AXIDUST }}$ observed during the soft state as SOFTABS.

\subsection{XMM-Newton hard state}

We performed a simultaneous fit of the seven new hard-state $X M M$ Newton spectra. The absorbed power-law model provides a superior fit ( $\chi_{\mathrm{PL}}^{2}=4155.7$ for 4046 dof), compared to either an absorbed blackbody ( $\chi_{\mathrm{BB}}^{2}=4541.7$ for 4046 dof $)$ or an absorbed disc blackbody $\left(\chi_{\mathrm{BB}}^{2}=4259.9\right.$ for $\left.4046 \mathrm{dof}\right)$.

Despite no clear residuals are observed in the Fe $\mathrm{K}$ band (see Fig. 5), we add the same ionized absorption component, with the best-fitting parameters observed during the soft state. This results in a significant worsening of the fit $\left(\Delta \chi^{2}=-17.6\right.$ for the same dof). We further check for the presence of ionized Fe K lines. No narrow absorption lines are detected at more than $\sim 95$ per cent significance (a narrow Fe XXVI absorption line is detected at $\sim 93$ percent and $\sim 90$ percent significance, during observation 0743630201 and during 0723410401, with an equivalent width $\mathrm{EW} \simeq-16$ and $\mathrm{EW} \simeq-13 \mathrm{eV}$, respectively, while we note that in the soft state such lines are typically observed with an equivalent width of $\mathrm{EW} \simeq-25 \mathrm{eV}, \mathrm{EW} \simeq-30 \mathrm{eV}$ ).

\footnotetext{
${ }^{3}$ Dips have been filtered out in the analysis presented in this paper (see Section 2.1).

${ }^{4}$ The apparently different best-fitting column density of neutral absorption obtained fitting the new data, compared to archival observations, is due to the different absorption models (e.g. here the TBABS instead than the PHABS model is used), to the assumed abundances and cross-sections as well as an improved treatment of the effects of dust scattering (see Ponti et al. 2017 for a more detailed description).
}

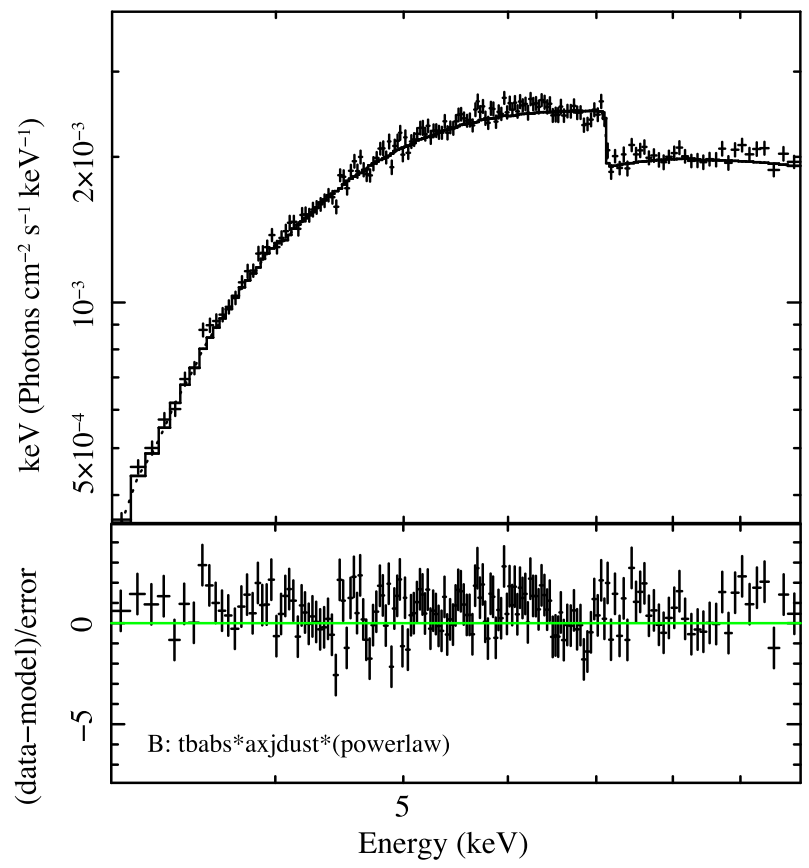

Figure 5. XMM-Newton combined spectrum of the seven new hard-state observations (Table A1). Top panel: Unfolded spectrum, fitted with the best-fitting model, composed by a power law absorbed by neutral material (TBABS*AXJDUST*(POWERLAW)). Bottom panel: Residuals, after fitting the data with the best-fitting model (TBABS*AXJDUST*(POWERLAW)).

From the spectra of the new hard-state observations, we can derive upper limits to the presence of narrow Fe Xxv and Fe XxvI $\mathrm{K} \alpha$ absorption lines between $\mathrm{EW}_{\mathrm{Fexxv}}>-15$ and $\mathrm{EW}_{\mathrm{Fexxv}}>-30 \mathrm{eV}$ and $\mathrm{EW}_{\mathrm{Fexxvi}}>-15$ and $\mathrm{EW}_{\mathrm{Fexxvi}}>-35 \mathrm{eV}$, respectively). This rules out the presence of the same ionized absorption component in the hard state, ubiquitously observed during the soft state. However, because of the shorter exposure, we note that these upper limits are less stringent than those obtained from the longer exposure of the 2014 April 03 observation (see Ponti et al. 2015a). Therefore, we cannot exclude the presence of absorption lines with EW of $-5:-15 \mathrm{eV}$ in these new observations.

We also tested that the addition of either an $\mathrm{Fe} \mathrm{K} \alpha$ disc line or Gaussian emission line does not improve significantly the fit ( $\chi^{2}=4153.9$ for 4038 dof), with upper limits to its equivalent width (assuming $\sigma=0.1 \mathrm{keV}$ ) of the order of $\mathrm{EW}<30 \mathrm{eV}$.

As observed in previous hard-state observations, the neutral absorption column density is consistent with being constant between the various hard-state observations (Table 1; $\Delta \chi^{2}=-8.9$ for the elimination of 6 dof). We also note that the best-fitting equivalent neutral hydrogen column density is $N_{\mathrm{H}}=(28.9 \pm 0.5) \times 10^{22} \mathrm{~cm}^{-2}$, consistent with the value observed during the soft state, when fitted with the disc blackbody component (see Table 1 and Ponti et al. 2015a).

Hereinafter, we will refer to the absorption and scattering components TBABS*AXJDUST observed during the hard state as HARDABS.

\section{SIMULTANEOUS XMM-NEWTON AND NUSTAR OBSERVATIONS}

Seven XMM-Newton observations are simultaneous to five NuSTAR ones (see Table A1 and A2). To achieve the best possible constraints on the evolution of the X-ray SED, we performed a combined fit of these simultaneous XMM-Newton and NUSTAR data. The joint 


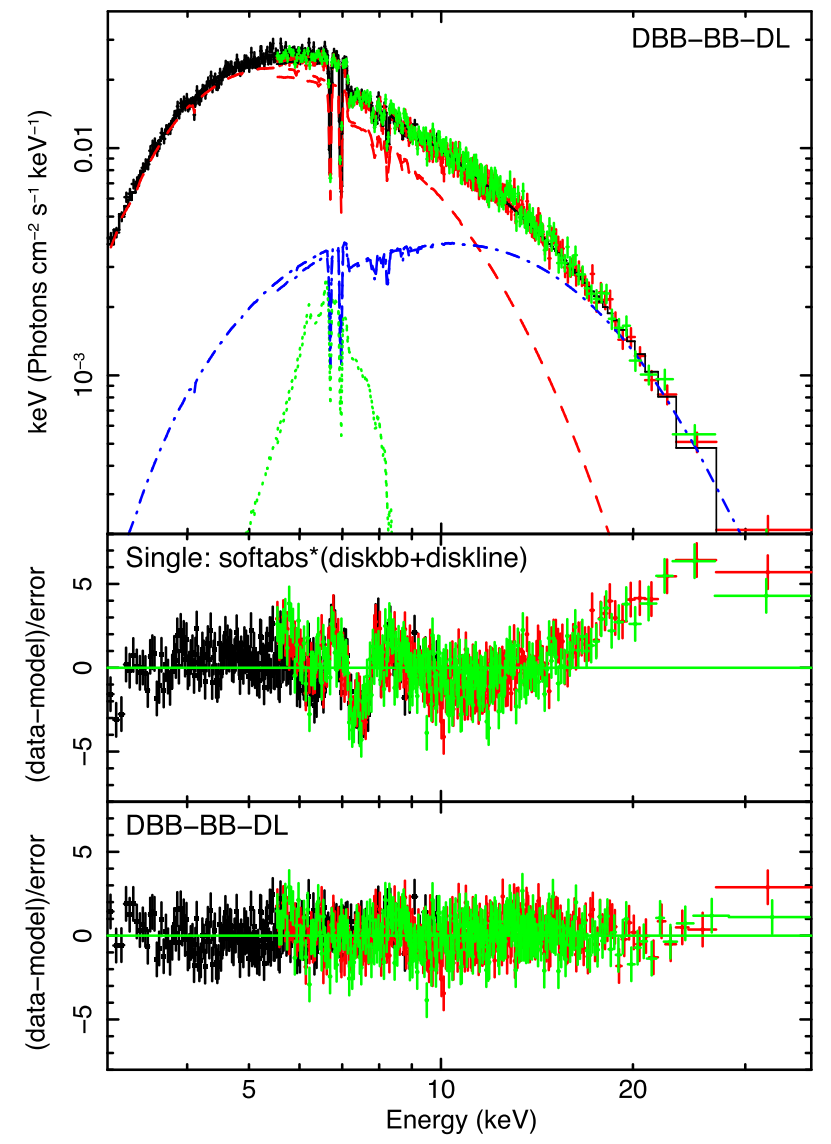

Figure 6. XMM-Newton + NuSTAR spectra of the longest simultaneous softstate observation obtained on (2015 February 25 and 26). Upper panel: Broad-band fit performed with the double thermal plus disc-line model (DBB-BB-DL). The black, red and green data show the XMM-Newton, NuSTAR A and B modules, respectively. The dashed red, dash-dotted blue, dotted green and solid black lines show the emission from the disc blackbody, blackbody, disc-line components and total emission, respectively. Panel single: Residuals after fitting the spectra with the single thermal continuum model (softabs $*($ diskbb + diskline $)$ ) best-fitting the soft-state XMM-Newton spectra. Clear residuals are left at high energy, implying the need for a second continuum component. Lower panel: Residuals left by the fit with an absorbed double thermal component model (DBB-BB-DL) imposing a hotter disc blackbody component than the blackbody one. The model provide an acceptable description of the data.

XMM-Newton and NUSTAR fit revealed a discrepancy at low energy. Full details and discussion about this discrepancy are provided in Appendix A.

\subsection{NuSTAR+XMM-Newton soft state: longest observation on 2015 February 26}

We started by fitting the longest simultaneous XMM-Newton plus NuSTAR observation that caught AX J1745.6-2901 in the soft state, fitting the low energy excess with the modified absorption model (Section A3). We initially employed the single emission component model used for the XMM-Newton data (Section 4.1). Therefore, we fitted the spectra with an absorbed disc blackbody plus disc line model (SOFTABS*(DISKBB+DISKLINE)). This fit left large residuals (see panel 'single' of Fig. 6) at high energies, producing an unacceptable fit $\left(\chi^{2}=2225.6\right.$ for 1362 dof). These residuals demonstrated the need for a second broad-band spectral emission component.

\subsubsection{Two thermal emission components plus disc line}

We then added to the model an extra blackbody component (SOFTABS*(DISKBB+BBODY+DISKLINE), dubbed DBB-BB-DL), producing a significant improvement of the fit $\left(\Delta \chi^{2}=881.7\right.$ for two new free parameters; $\chi^{2}=1343.9$ for 1360 dof). With this model, the spectra are best fit by a prominent and hot $\left(k T_{\mathrm{BB}} \sim 1.19 \pm 0.03 \mathrm{keV}\right)$ blackbody component produced from a surface with a reasonable (however small) radius of $r_{\mathrm{BB}}=3.0 \pm 0.2 \mathrm{~km}$. However, the highenergy emission is produced by a very hot disc blackbody emission $\left(k T_{\mathrm{DBB}}=3.4 \pm 0.1 \mathrm{keV}\right)$ with an inner radius of the accretion disc $\left(r_{\mathrm{DBB}} \sim 1 \mathrm{~km}\right)$ significantly smaller than the NS radius. For this reason, this result is unphysical and it will not be discussed any longer.

We then fitted the spectrum with the same model, but imposing the temperature of the disc blackbody emission to be smaller than the one of the blackbody (Fig. 6; Table 2). With this setup, the spectra were fit by a warm $\left(k T_{\mathrm{DBB}}=1.77 \pm 0.05 \mathrm{keV}\right)$ disc blackbody component that reproduced most of the observed $\mathrm{X}$-ray emission. In particular, the best-fitting inner disc radius is several times larger than in the previous case $\left(r_{\mathrm{DBB}}=5.7 \pm 0.4\right.$ $\mathrm{km})$, however still rather small. This time, the blackbody components appeared hotter $\left(k T_{\mathrm{BB}}=3.0 \pm 0.1 \mathrm{keV}\right)$ and smaller $\left(r_{\mathrm{BB}} \sim 0.28 \pm 0.04 \mathrm{~km}\right)$. Therefore, despite this set-up provides a slightly worse fit $\left(\Delta \chi^{2}=-4.6\right)$ compared to the previous set-up, we preferred it on physical grounds.

\subsubsection{Thermal plus Comptonization and disc-line components}

Alternatively, the high energy part of the emission of AX J1745.62901 could be produced by Comptonization.

\section{High-energy cut-off in the X-ray band (SIMPL)}

To start, we parametrized the Comptonization component by substituting the hot blackbody emission with a Comptonized radiation model that assumes that the high-energy cut-off is at very high energy (the SIMPL convolution model; Steiner et al. 2009). Therefore, we fitted the data with the model SIMPDBB-DL: SOFTABS*(SIMPL(DISKBB)+DISKLINE). This model provided a significantly worse fit compared to the previous models $\left(\chi^{2}=1397.2\right.$ for 1360 dof; Table 2$)$.

We note that the photon index of the Comptonized component yields an excessively steep value of $\Gamma=4.6 \pm 0.1$. Such steep spectra are typically signalling that either the Comptonized component has a thermal origin, or it indicates the presence of a high-energy cut-off in the power-law shape. To test this latter possibility, we refitted the spectrum assuming a photon index of $\Gamma=2$ for the Comptonized component and we added to the model an exponential cutoff $^{5}$ (HIGHECUT in XSPEC; SOFTABS*SIMPL(DISKBB+DISKLINE)HIGHECUT). We obtained a comparable fit $\left(\Delta \chi^{2}=5.3\right.$ for the same dof $)$ for a cut-off energy of $E_{c}=6.7 \pm 0.1 \mathrm{keV}$. This indicates that if the high-energy emission is due to a Comptonization component, then the Comptonizing electrons must have a temperature of a few $\mathrm{keV}$, therefore about two orders of magnitude lower than what is observed in $\mathrm{BH}$ binaries and NS during the hard state (see Section 5.3).

\footnotetext{
${ }^{5}$ We assumed the folded energy to be equal to the cut-off energy.
} 


\section{Comptonization with high-energy cut-off (NTНСОМP)}

We then applied a more sophisticated Comptonization model that self-consistently reproduces both the power-law shape and the highenergy cut-off (NTHCOMP; Zdziarski, Johnson \& Magdziarz 1996; Życki, Done \& Smith 1999). Therefore, we applied the model SOFTABS* (DISKBB+NTHCOMP+DISKLINE) that we call DBB-NTH-DL. We assumed the temperature of the seed Comptonized photons to be equal to the temperature of the disc blackbody component.

This provides a good fit $\left(\chi_{\text {DBB }}^{2}=1346.9\right.$ for 1359 dof; see Table 2), slightly better than the double thermal emission model. In particular, we now observe that the asymptotic power-law photon index is steep $(\Gamma \sim 2.2)$, however physically acceptable and within the range of values observed during the soft state in other NS and $\mathrm{BH}$ systems. Moreover, the temperature of the Comptonizing electron is constrained to be $k T_{\mathrm{e}} \sim 3-5 \mathrm{keV}$, therefore producing a highenergy cut-off in the X-ray band, as also suggested by the fit with the SIMPL component. On the other hand, we note that the derived inner disc radius appears to be too small $\left(r_{\mathrm{DBB}}=5.5 \pm 0.8 \mathrm{~km}\right)$, even considering the large uncertainties associated with the derivation of this parameter, unless AX J1745.6-2901 is beyond $16 \mathrm{kpc}$ from us.

We also consider the alternative scenario where the thermal emission is produced by blackbody emission and not by the disc blackbody. We call this model BB-NTH-DL, SOFTABS* (BBODY+NTHCOMP+DISKLINE). We note that this model can also reproduce the data $\left(\chi_{\mathrm{BB}}^{2}=1344.1\right.$ for 1359 dof; Table 2$)$.

\subsubsection{Two thermal components plus relativistic ionized reflection}

The simultaneous XMM-Newton $+N u S T A R$ spectra are consistent with the presence of a broad $\mathrm{Fe} \mathrm{K} \alpha$ emission line during this softstate observation. The $\mathrm{Fe} \mathrm{K} \alpha$ line is often the most prominent feature of a reflection component (Fabian et al. 2000, 2009; Nandra et al. 2007; Ponti et al. 2006, 2010; Plant et al. 2014). We, therefore, tested for the presence of such a reflection component by substituting the DISKLINE with a BBREFL model. The BBREFL model reproduces a self-consistent ionized reflection spectrum obtained by illuminating a constant density slab with a blackbody spectrum (Ballantyne 2004). We convolved the BBREFL ionized reflection component with the KDBLUR kernel, that is mimicking the relativistic effects on the shape of the reflection component off an accretion disc around a compact source (Laor 1991). We assumed a disc inclination, inner and outer radii of $80^{\circ}, 6 r_{g}$ and $400 r_{g}$, respectively. We also fixed the value of the emissivity index to $\alpha=2.4$, such as derived by the fit with the disc line component, the Iron abundance to Solar and ionization parameter to $\log (\xi)=1$.

We fitted the double thermal plus relativistic ionized reflection model to the data: SOFTABS* (DISKBB+BBODY+KDBLUR(BBREFL)). We call this model, DBB-BB-RR. Because the disc blackbody component is dominating the source emission up to $\sim 15 \mathrm{keV}$, we imposed that the temperature of the illuminating blackbody emission is equal to the temperature of the disc blackbody component. This model provided a significant improvement of the fit, compared to any previously considered model $\left(\chi^{2}=1318.3\right.$ for 1360 dof; see Fig. 7 and Table 2$)$. The introduction of the ionized disc reflection component (green dotted line in Fig. 7) allowed us to reproduce the broad excess in the Fe $\mathrm{K}$ band well. Additionally, we note that the spectrum can be fitted with a cooler disc blackbody $\left(k T_{\mathrm{DBB}}=1.55 \pm 0.11 \mathrm{keV}\right)$, with a slightly larger inner disc radius of $r_{\mathrm{DBB}}=7.5 \pm 1.1 \mathrm{~km}$. Moreover, because of the Compton reflection hump, the reflection component has a harder spectrum in the $10-40 \mathrm{keV}$ band compared

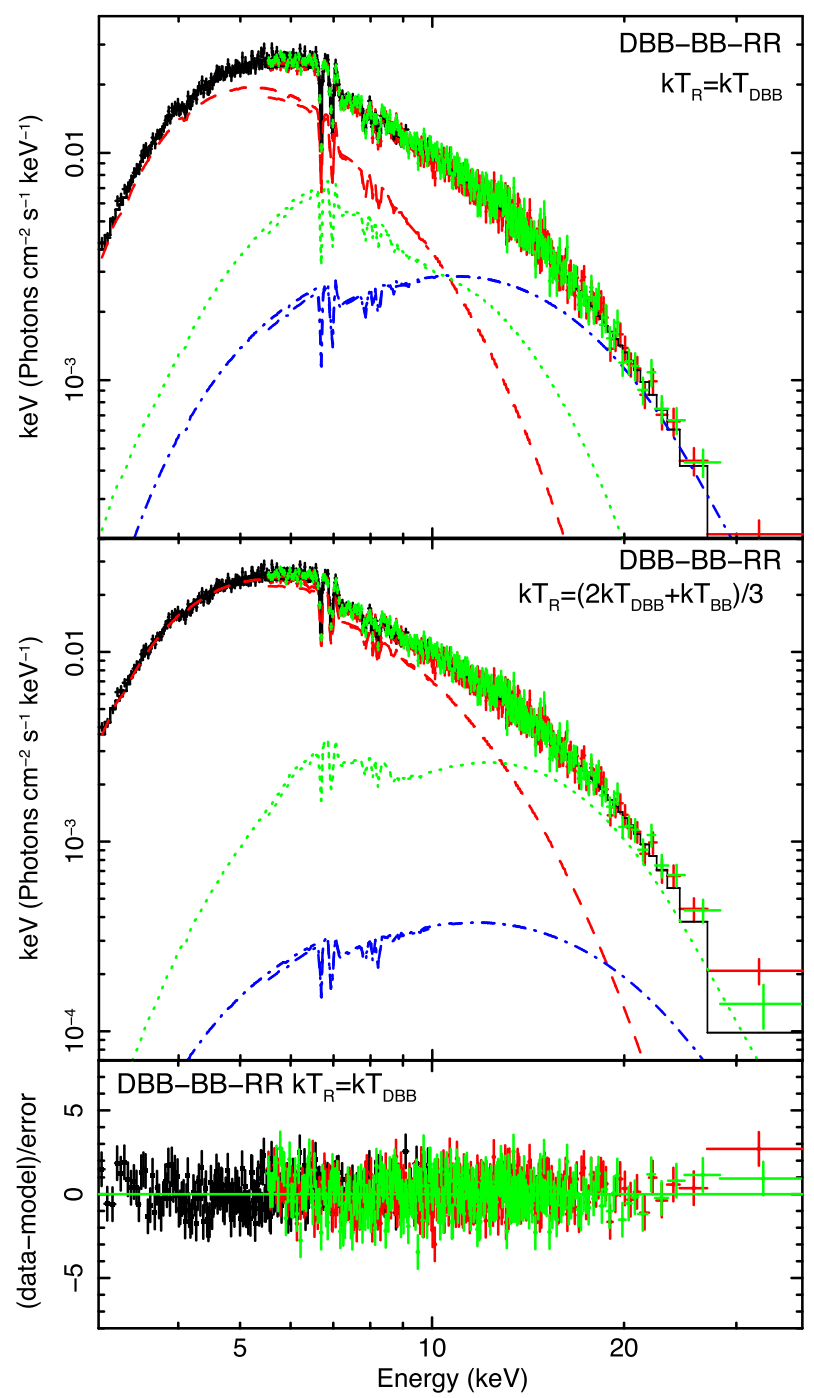

Figure 7. XMM-Newton $+N u S T A R$ spectra of the longest simultaneous softstate observation, same as Fig. 6. Upper panel: Best-fitting broad-band fit performed with the double thermal plus relativistic ionized reflection model (DBB-BB-RR). The dashed red, dash-dotted blue, dotted green and solid black lines show the emission from the disc blackbody, blackbody, relativistically ionized reflection components and total emission, respectively. The reflection spectrum is produced by illuminating the disc with a thermal emission with $k T_{\mathrm{R}}=k T_{\mathrm{DBB}}$. Middle panel: Same as upper panel, but assuming that the disc is irradiated by a hotter thermal component $\left(k T_{\mathrm{R}}=\left(2 \times k T_{\mathrm{DBB}}+k T_{\mathrm{BB}}\right) / 3\right)$. Lower panel: Residual left once the data are fitted with the model shown in the upper panel.

to the illuminating source. This allowed us to reproduce part of the high energy emission in excess above the extrapolation of the disc blackbody component (see Fig. 7). However, by fixing the temperature of the illuminating blackbody to the temperature of the disc blackbody, the ionized reflection could not reproduce all of the high energy emission, therefore it was still requiring the presence of a hot $\left(k T_{\mathrm{BB}}=3.1 \pm 0.2 \mathrm{keV}\right)$ blackbody emission from small patches on the NS surface $\left(r_{\mathrm{BB}} \sim 0.2-0.3 \mathrm{~km}\right)$.

We noted that the hot blackbody component could also contribute to the disc irradiation. If so, the effective temperature of the thermal emission irradiating the disc and producing the reflection spectrum could be higher than $k T_{\mathrm{DBB}}$ (as assumed before). We therefore re-fitted the spectra with the same model, but assuming 


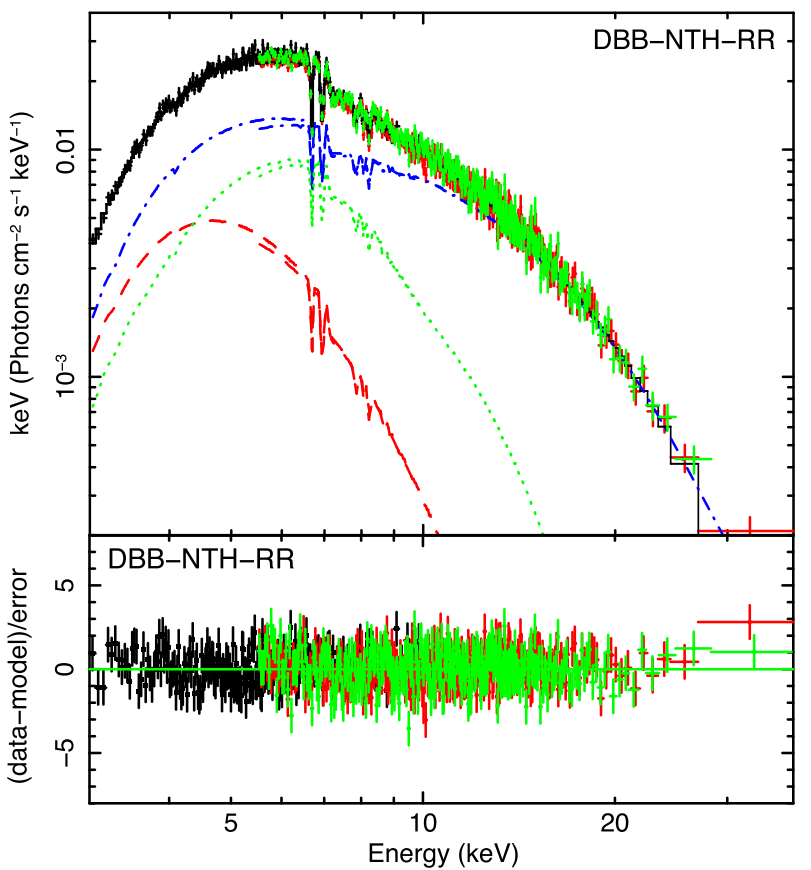

Figure 8. XMM-Newton + NUSTAR spectra of the longest simultaneous softstate observation (same as Fig. 6), once fitted with the model DBB-NTHRR. The disc blackbody, Comptonization (NTHCOMP) and ionized reflection (BBREFL) are shown with red dashed, blue dot-dashed and green dotted lines, respectively.

that the reflection is produced by a blackbody irradiation with temperature $k T_{\mathrm{R}}=k\left(2 \times T_{\mathrm{DBB}}+T_{\mathrm{BB}}\right) / 3$, intermediate between the temperature of the warm disc blackbody and hot blackbody one. The model, with these assumptions provided a significant worsening of the fit $\left(\Delta \chi^{2}=-30.2\right.$ for the same dof). The central panel of Fig. 7 shows that, as expected, the reflection spectrum (green dotted line) is indeed harder than before. In particular, the spectral shape of the reflection component is similar to the one of the hot blackbody component (blue dash-dotted line). Such a hard reflection spectrum can therefore reproduce the majority of the hard X-ray emission, therefore it appears to be several times brighter than the hot blackbody emission. This seems physically unlikely. For this reason, we disregard this model and hereinafter we will assume $k T_{\mathrm{R}}=k T_{\mathrm{DBB}}$ (or $k T_{\mathrm{R}}=k T_{\mathrm{BB}}$ ).

\subsubsection{Thermal plus Comptonization and relativistic reflection}

We then employed the same model, explored in section 5.1.2, but we exchanged the DISKLINE component with a relativistic ionized reflection one, i.e. SOFTABS*(DISKBB+NTHCOMP+KDBLUR(BBREFL)) and SOFTABS $*($ BBODY +NTHCOMP+KDBLUR(BBREFL)) and we called these DBB-NTH-RR and BB-NTH-RR, respectively (Table 2 and Fig. 8). We note that in all cases, the models involving the relativistic ionized reflection component provided a significantly better description of the data, compared to the ones employing a disc line (Table 2). Moreover, model DBB-NTH-RR appears preferred over the BB-NTH-RR one $\left(\Delta \chi^{2}=21.3\right.$ for the same dof $)$.

The model DBB-NTH-RR provided a good description of the data with reasonable best-fitting parameters. In particular, we note that the temperature of the disc blackbody component is significantly lower $\left(k T_{\mathrm{DBB}}=1.1 \pm 0.1 \mathrm{keV}\right)$ and the inner disc radius $\left(r_{\mathrm{DBB}} \sim 9 \pm 3 \mathrm{~km}\right.$ ) larger than with the other models considered.

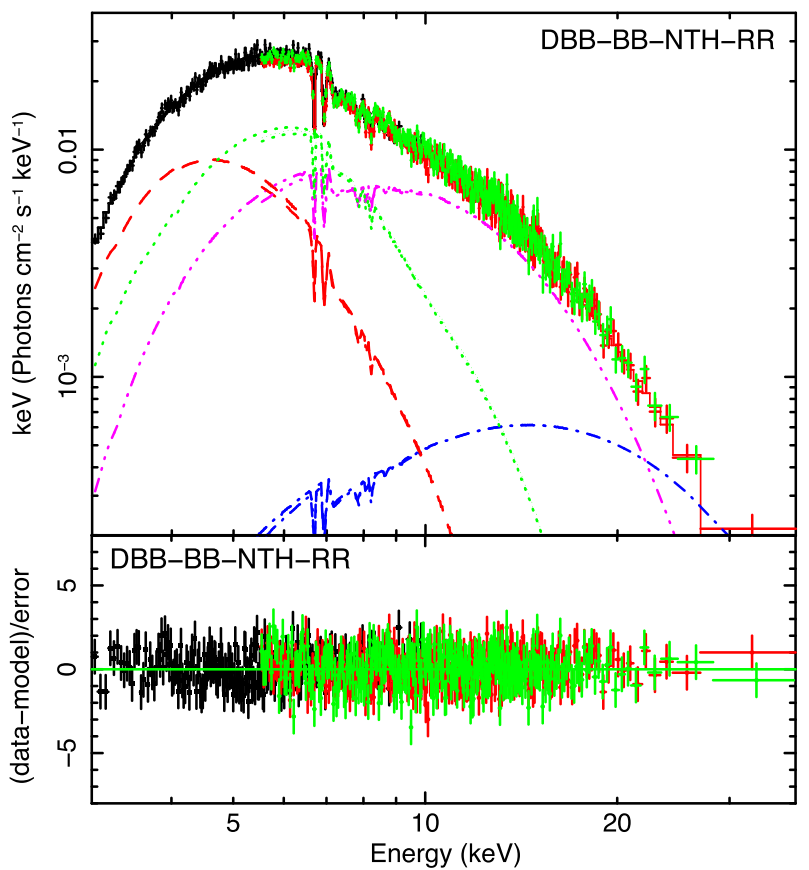

Figure 9. XMM-Newton + NUSTAR spectra of the longest simultaneous softstate observation (same as Fig. 6), once fitted with the three component model DBB-BB-NTH-RR. The disc blackbody, blackbody, Comptonization (NTHCOMP) and relativistic ionized reflection (BBREFL) are shown with red dashed, magenta dot-dot-dashed, blue dot-dashed and green dotted lines, respectively.

Indeed, the hard X-ray flux is now reproduced by the combination of the harder reflection and Comptonization components (see Fig. 8), requiring no strong contribution from the soft disc blackbody component. We observed that the un-absorbed flux of the disc blackbody emission in the $1-500 \mathrm{keV}$ band is $F_{1-500} \sim 1.8 \times 10^{-10} \mathrm{erg}$ $\mathrm{cm}^{-2} \mathrm{~s}^{-1}$ about half the flux of the Comptonization component $F_{1-500} \sim 3.7 \times 10^{-10} \mathrm{erg} \mathrm{cm}^{-2} \mathrm{~s}^{-1}$, while the flux of the ionized reflection component is rather high being $F_{1-500} \sim 1.5 \times 10^{-10} \mathrm{erg}$ $\mathrm{cm}^{-2} \mathrm{~s}^{-1}, \sim 27$ per cent of the total illuminating flux.

\subsubsection{Three components model}

We finally fit the spectrum with the three components model (DBB-BB-NTHCOMP-RR), composed by the double thermal plus Comptonization and relativistic ionized reflection (SOFTABS* (DISKBB +BBODY+NTHCOMP+KDBLUR(BBREFL)) $)$. This model provides a significant improvement of the fit compared to previously tested ones $\left(\Delta \chi^{2}=18.5\right.$ for the addition of two parameters, $F$-test probability $\left.\sim 8 \times 10^{-5}\right)$.

With this model, the soft X-ray emission is dominated by a warm $k T_{\mathrm{DBB}}=1.1 \pm 0.1 \mathrm{keV}$ disc blackbody component with a reasonable inner disc radius of $r_{\mathrm{DBB}}=14 \pm 2 \mathrm{~km}$, corresponding to $\sim 7 r_{g}$, therefore larger than the NS radius. A hotter blackbody component $\left(k T_{\mathrm{BB}}=2.3 \pm 0.2 \mathrm{keV}\right)$ emitted from a small area with a radius $r_{\mathrm{BB}}=0.6 \pm 0.2 \mathrm{~km}$ that dominates the $\sim 8-20 \mathrm{keV}$ band, while a faint and hard $(\Gamma \sim 1-2.2)$ Comptonization component (with highenergy cut-off at $k T_{\mathrm{e}} \sim 5 \mathrm{keV}$ ) becomes important above $\sim 20 \mathrm{keV}$ (see Fig. 9).

We observed that the un-absorbed flux of the disc blackbody emission in the $1-500 \mathrm{keV}$ band is $F_{1-500} \sim 3.9 \times 10^{-10} \mathrm{erg}$ $\mathrm{cm}^{-2} \mathrm{~s}^{-1}$. A smaller, however, comparable flux is observed to be produced by blackbody radiation $F_{1-500} \sim 2.0 \times 10^{-10} \mathrm{erg}$ 
$\mathrm{cm}^{-2} \mathrm{~s}^{-1}$, while the Comptonization component carries only a small fraction of the energy $F_{1-500} \sim 0.9 \times 10^{-10} \mathrm{erg} \mathrm{cm}^{-2} \mathrm{~s}^{-1}$. We note that the flux of the ionized reflection component is high $F_{1-500} \sim 2.3 \times 10^{-10} \mathrm{erg} \mathrm{cm}^{-2} \mathrm{~s}^{-1}$, corresponding to $\sim 30$ per cent of the total.

\subsection{NuSTAR+XMM-Newton soft state: on 2015 April 01 and 2015 April 02}

We then applied the same models best fitting the longest simultaneous XMM-Newton+NuSTAR observation (DBB-BB-DL, DBB-NTH-RR, BB-NTH-RR and DBB-BB-NTH-RR) to the other two soft state simultaneous XMM-Newton plus NuSTAR observations. The statistics of these spectra is slightly lower. For this reason, the parameters (e.g. photon index and electron energy) of the weak Comptonized component cannot be well constrained. Therefore, we fitted these data assuming the best-fitting values observed during the 2015 February 25 observation $\left(\Gamma=1.1\right.$ and $\left.k T_{\mathrm{e}}=4.6 \mathrm{keV}\right)$.

The fit of these additional two XMM-Newton+NuSTAR spectra produced results similar to what was observed during the longer observation on 2015 February 26. Indeed, the spectra were best reproduced by the three component model (DBB-BB-NTH-RR). We also observed that the best-fitting parameters were consistent with the ones obtained during the 2015 February 25 observation (Table 3 ).

The best-fitting unabsorbed flux of the disc blackbody, blackbody and Comptonized emission during the observation accumulated on 2015 April 02 were $F_{1-200} \sim 4.4 \times 10^{-10}, \sim 1.9 \times 10^{-10}$ and $\sim 0.1 \times 10^{-10} \mathrm{erg} \mathrm{cm}^{-2} \mathrm{~s}^{-1}$, respectively. The flux of the ionized reflection component was $\sim 25$ percent of the total. Instead, on 2015 April 02 they were $F_{1-200} \sim 3.2 \times 10^{-10}, \sim 2.7 \times 10^{-10}$ and $\sim 0.7 \times 10^{-10} \mathrm{erg} \mathrm{cm}^{-2} \mathrm{~s}^{-1}$, respectively. The flux of the ionized reflection component was $\sim 33$ per cent of the total.

\subsection{NuSTAR+XMM-Newton hard state}

We then fitted the two hard-state contemporaneous XMM-Newton plus NUSTAR spectra (Table 4). Each of the two long NuSTAR exposures (which started on 2014 August 30 and on 2014 September 27) was partially covered by two shorter XMM-Newton observations (Table A1). For this reason, we fitted the four spectra (2XMMNewton plus NUSTAR FPMA and FPMB) together. We observed no major spectral variation between the XMM-Newton and $N u S$ $T A R$ observations; therefore, we employed the same model, with the same parameters, to fit the four spectra. We only allowed a normalization constant to be different between the various spectra (in order to reproduce small normalization differences, associated with minor source flux variations; see Table 4).

We started by fitting the XMM-Newton plus NUSTAR spectra with the absorbed power-law model best fitting the XMM-Newton spectra (Section 4.2). This simple model very well reproduces the X-ray emission of AX J1745.6-2901, during the hard state. The observed power-law photon index $(\Gamma=1.86-1.88)$ is, in all cases, within the range of values typically observed during the hard state. The inter normalization constants are within $\sim 10$ percent of the expected value, indicating a minor flux variation of the source between the different spectra.

As typically observed in accreting BH and NS, the disc blackbody emission is far less prominent during the hard state than in the soft (Done et al. 2007; Dunn et al. 2010). In fact, during hard states, the thermal emission is commonly weaker and far colder than during the soft state, with observed temperatures in the range: $k T \sim 0.2-0.7 \mathrm{keV}$ (Dunn et al. 2010). Being AX J1745.6-2901 highly absorbed, we cannot detect any X-ray photon below $\sim 3 \mathrm{keV}$, preventing us from placing strong constraints on the temperature of such thermal component during the hard state. On the other hand, to reconstruct fiducial source SED, it is essential to constrain the position and intensity of the disc emission. Therefore, to restrain this, we fitted the spectra with an array of models containing a soft thermal component, in addition to the Comptonization one, producing the power-law emission. In particular, we employed the models SIMPDBB and SIMPBB that are reminiscent of the same models that we used for the soft state, once removing the disc-line and ionized absorption components (resulting in the models: HARDABS*(SIMPL*DISKBB)) and HARDABS*(SIMPL*BBODY), respectively). For each of these models, we explored an array of possible parameters, such as disc blackbody temperatures and/or Comptonization fractions ${ }^{6}$ (Table 4).

We observed that any disc blackbody component with temperature in the range $k T_{\mathrm{DBB}} \sim 0.2-0.7 \mathrm{keV}$ and Comptonization fractions within $f_{\mathrm{sc}} \sim 0.3-1.0$ are consistent with the data, while hotter $k T_{\mathrm{DBB}} \sim 1.0 \mathrm{keV}$ ( or $k T_{\mathrm{BB}} \sim 0.7 \mathrm{keV}$ ) thermal emission produces a significantly worse fit (Table 4$)$.

To constrain the presence of a high-energy cut-off, we also employed the model DBB-NTH (implemented in XSPEC as HARDABS*(DISKBB+NTHCOMP)). For both series of data sets, we observed either better or comparable results whenever the disc blackbody temperature is assumed to be in the range of $k T_{\mathrm{DBB}} \sim 0.2-$ $0.7 \mathrm{keV}$ (Table 4). For disc temperatures significantly higher than $k T_{\mathrm{DBB}}=0.7 \mathrm{keV}$, the inner disc radius results to be smaller than the NS radius; therefore, we can rule this out as unphysical. On the other hand, smaller disc temperatures are possible and they imply only upper limits to the inner disc radius (e.g. for $k T_{\mathrm{DBB}}=0.2 \mathrm{keV}$, $r_{\text {DBB }}<10^{3} \mathrm{~km}$; Table 4 ). No high-energy cut-off is detected within the observed X-ray band. Indeed, only lower limits to the temperature of the Comptonizing electrons are observed $\left(k T_{\mathrm{e}}>70\right.$ $100 \mathrm{keV}$; Table 4). This appears very different to what is observed during the soft state, when the cut-off is at $k T_{\mathrm{e}} \sim 3-5 \mathrm{keV}$ (Section 5.1). We conclude by observing that this simplified model can reproduce the observed X-ray spectra during the hard state of AX J1745.6-2901.

\section{FIT OF THE REMAINING NUSTAR OBSERVATIONS}

We then fitted all soft-state NUSTAR only observations with the bestfitting three component model DBB-BB-NTH-RR. Because of the lower statistics (compared to the simultaneous XMM-Newton+NuSTAR fits), we fixed the equivalent hydrogen column density, the column density and ionization parameter of the ionized absorber as well as the photon index and electron temperature of the Comptonization component to the best-fitting values observed during the 2015 February 25 observation $\left(N_{\mathrm{H}}=32 \times 10^{22} \mathrm{~cm}^{-2}, \log \left(\xi_{\mathrm{IA}}\right)=4.1\right.$, $\log \left(N_{H_{\mathrm{IA}}}\right)=23.5, \Gamma=1.1$ and $\left.k T_{\mathrm{e}}=4.5 \mathrm{keV}\right)$. We observed that this model always provides a good description of the data, with bestfitting parameters similar to the one derived from the simultaneous $X M M$-Newton + NuSTAR observations (Table 5).

We also modelled the hard-state NUSTAR only observation with the model DBB-NTH, also in this case obtaining acceptable fits and reasonable best-fitting parameters (see Table 5).

\footnotetext{
${ }^{6}$ We define as Comptonization fraction the fraction of seed photons that scatter producing inverse Compton radiation (such as defined in the SIMPL model; Steiner et al. 2009).
} 
Table 4. Best-fitting parameters for the two hard state simultaneous XMM-Newton+NuSTAR observations accumulated on 2014 September 28 and 2014 August 31. $c_{\mathrm{xmm} 2}$ shows the normalization constant of the second XMM-Newton spectrum. See as caption of Table 2 for a description of the other parameters. ${ }^{\star}$ Indicated the parameter is fixed to the given value. For model SIMPDBB, we report the variation of the best-fitting parameters to the assumed parameters. For the observation accumulated on 2014 September 28. For example, assuming $F_{\mathrm{sc}}=1$ and $k T_{\mathrm{DBB}}=0.4,0.7$ and $1.0 \mathrm{keV}$, we obtained $N_{\mathrm{DBB}}=46 \pm 2,5.1 \pm 0.1$ and $1.27 \pm 0.03$, with $\chi^{2} /$ dof $=2361.5 / 2220,2363.2 / 2220$ and 2385.9/2220, respectively (all other parameters of the model do not vary significantly), while assuming $k T_{\mathrm{DBB}}=0.4 \mathrm{keV}$ and $F_{\mathrm{sc}}=0.6$ and 0.3 , we obtained $N_{\mathrm{DBB}}=78 \pm 3$ and $160 \pm 5$, with $\chi^{2} /$ dof $=2362.5 / 2220$ and $2366.2 / 2220$. We then performed the same exercise for model sIMPBB. For $k T_{\mathrm{BB}}=0.7$ and $F_{\mathrm{sc}}=1.0$, we obtain $N_{\mathrm{BB}}=10.9 \pm 0.2$ and $\chi^{2} / \mathrm{dof}=2383.4 / 2220$, while for $k T_{\mathrm{BB}}=0.4$ and $F_{\mathrm{sc}}=0.3$, we obtain $N_{\mathrm{BB}}=362 \pm 8$ and $\chi^{2} / \mathrm{dof}=2381.0 / 2220$. For the observation accumulated on 2014 August 31 . For example, assuming $F_{\mathrm{sc}}=1$ and $k T_{\mathrm{DBB}}=0.4,0.7$ and $1.0 \mathrm{keV}$, we obtained $N_{\mathrm{DBB}}=44 \pm 2,4.8 \pm 0.1$ and $1.19 \pm 0.03$, with $\chi^{2} / \mathrm{dof}=2186.6 / 2130,2188.1 / 2130$ and 2214.0/2130, respectively (all other parameters of the model do not vary significantly), while assuming $k T_{\mathrm{DBB}}=0.4 \mathrm{keV}$ and $F_{\mathrm{sc}}=0.6$ and 0.3 , we obtained $N_{\mathrm{DBB}}=74 \pm 3$ and $150 \pm 6$, with $\chi^{2} / \mathrm{dof}=2188.8 / 2130$ and $2194.9 / 2130$. We then performed the same exercise for model simpBB. For $k T_{\mathrm{BB}}=0.7$ and $F_{\mathrm{sc}}=1.0$, we obtain $N_{\mathrm{BB}}=10.0 \pm 0.2$ and $\chi^{2} / \mathrm{dof}=2209.7 / 2130$, while for $k T_{\mathrm{BB}}=0.4$ and $F_{\mathrm{sc}}=0.3$, we obtain $N_{\mathrm{BB}}=340 \pm 9$ and $\chi^{2} /$ dof $=2210.6 / 2130$.

\begin{tabular}{|c|c|c|c|c|c|}
\hline \multicolumn{6}{|c|}{ Simultaneous XMM-Newton + NuSTAR hard state (2014 September 28) } \\
\hline Model & PL & SIMPDBB & SIMPBB & & \\
\hline$N_{\mathrm{H}}$ & $28.5 \pm 1.2$ & $28.8 \pm 1.3$ & - & $27.8 \pm 1.2$ & $30.5 \pm 2.0$ \\
\hline$k T_{\mathrm{DBB}}$ & - & $0.2^{\star}$ & - & 0.2 & 0.7 \\
\hline$N_{\text {DBB }}$ & - & $720 \pm 30$ & - & $<170000$ & $6 \pm 3$ \\
\hline$k T_{\mathrm{BB}}$ & - & - & $0.4^{\star}$ & - & - \\
\hline$N_{\mathrm{BB}}$ & - & - & $97 \pm 2$ & - & - \\
\hline$\Gamma$ & $1.83 \pm 0.05$ & $1.84 \pm 0.03$ & - & $1.84 \pm 0.02$ & $1.84 \pm 0.02$ \\
\hline$N$ & $1.34 \pm 0.08$ & - & - & - & - \\
\hline$F_{\mathrm{sc}}$ & - & $1.0^{\star}$ & $1.0^{\star}$ & - & - \\
\hline$N_{\mathrm{Nu}}$ & $1.3 \pm 0.1$ & - & - & - & - \\
\hline$k T_{\mathrm{e}}$ & - & - & - & $>120$ & $>100$ \\
\hline$N_{\text {nth }}$ & - & - & - & $1.29 \pm 0.07$ & $0.76 \pm 0.03$ \\
\hline$c_{\mathrm{xmm} 2}$ & $0.98 \pm 0.02$ & $0.97 \pm 0.01$ & - & $0.97 \pm 0.01$ & $0.97 \pm 0.02$ \\
\hline$c_{\mathrm{NuA}}$ & - & $0.95 \pm 0.03$ & - & $0.93 \pm 0.03$ & $0.91 \pm 0.03$ \\
\hline$c_{\mathrm{NuB}}$ & $1.10 \pm 0.02$ & $1.04 \pm 0.03$ & - & $1.02 \pm 0.03$ & $1.00 \pm 0.03$ \\
\hline$\chi^{2} /$ dof & $2360.2 / 2218$ & $2361.7 / 2220$ & $2360.7 / 2220$ & $2380.7 / 2218$ & $2354.9 / 2218$ \\
\hline
\end{tabular}

Simultaneous XMM-Newton+NuSTARhard state (2014 August 31)

\begin{tabular}{|c|c|c|c|c|c|}
\hline Model & PL & SIMPDBB & SIMPBB & & \\
\hline$N_{H}$ & $28.3 \pm 1.1$ & $28.1 \pm 1.2$ & - & $28.0 \pm 0.6$ & $31.4 \pm 2.5$ \\
\hline$k T_{D B B}$ & & $0.2^{\star}$ & & 0.2 & 0.7 \\
\hline$N_{D B B}$ & & $710 \pm 35$ & & $<160000$ & $9 \pm 4$ \\
\hline$k T_{B B}$ & & & $0.4^{\star}$ & & \\
\hline$N_{B B}$ & & & $90 \pm 2$ & & \\
\hline$\Gamma$ & $1.88 \pm 0.03$ & $1.88 \pm 0.03$ & - & $1.88 \pm 0.02$ & $1.87 \pm 0.02$ \\
\hline$N$ & $1.36 \pm 0.08$ & & & & \\
\hline$F_{s c}$ & & $1.0^{\star}$ & $1.0^{\star}$ & & \\
\hline$N_{N u}$ & $1.41 \pm 0.11$ & & & & \\
\hline$k T_{e}$ & & & & $>150$ & $>70$ \\
\hline$N_{n t h}$ & & & & $1.34 \pm 0.07$ & $0.72 \pm 0.03$ \\
\hline$c_{x m m 2}$ & $1.09 \pm 0.02$ & $1.09 \pm 0.02$ & - & $1.09 \pm 0.02$ & $1.09 \pm 0.02$ \\
\hline$c_{N u A}$ & & $1.02 \pm 0.03$ & - & $1.02 \pm 0.03$ & $1.02 \pm 0.03$ \\
\hline$c_{N u B}$ & $1.07 \pm 0.02$ & $1.09 \pm 0.03$ & - & $1.09 \pm 0.04$ & $1.08 \pm 0.03$ \\
\hline$\chi^{2} /$ dof & $2174.7 / 2130$ & $2187.1 / 2130$ & $2184.6 / 2130$ & $2191.2 / 2128$ & $2164.9 / 2128$ \\
\hline
\end{tabular}

\section{DISCUSSION}

We analysed the persistent emission from AX J1745.6-2901. Thanks to simultaneous NuSTAR+XMM-Newton observations, we could detail the emission mechanisms and obtain accurate X-ray SEDs for both the soft and hard states. We note that previous spectral fitting works on AX J1745.6-2901 were restricted to the limited energy band provided by XMM-Newton (Ponti et al. 2015a), therefore impeding detailed fits of the X-ray continuum with models more complex than a simple power law or (disc)blackbody emission for the hard and soft states, respectively. Thanks to the addition of the NuSTAR data, we demonstrated the richness of information of the X-ray emission from AX J1745.6-2901.
Strong spectral and flux variability is observed between the two states, on the other hand only moderate variations are observed between different observations within the same state.

All observations show clear evidence for a high column density of neutral absorption $\left(N_{\mathrm{H}} \sim 3.0 \times 10^{23} \mathrm{~cm}^{-2}\right)$. While it is well known that in dipping sources (during dips) the neutral absorption is highly variable (Frank, King \& Lasota 1987; Díaz Trigo et al. 2006; Ponti et al. 2016a), we observed that all soft- and hard-state spectra are roughly consistent with the same column density of absorbing material, once they are fitted with the same model (e.g. model DBB-BB-NTH-RR and SIMPBB). This is consistent with the idea that a significant fraction of the neutral absorption (during persistent emission) is produced by the interstellar medium (see Jin et al. 2017; Ponti et al. 2017). 
Table 5. Best-fitting parameters for the soft- and hard-state NuSTAR only observations. See as caption of Table 2 for a description of the various parameters. The ionization parameter and column density of the ionized absorption have been fixed to $\log \left(\xi_{\mathrm{IA}}\right)=4.1$ and $\log \left(N_{\mathrm{H}_{\mathrm{IA}}}\right)=23.5$, respectively. The column density of the neutral absorber is fixed to $N_{\mathrm{H}}=32 \times 10^{22} \mathrm{~cm}^{-2}$. The photon index and electron temperature of the Comptonized component are fixed to $\Gamma=1.1$ and $k T_{\mathrm{e}}=4.5 \mathrm{keV}$.

\begin{tabular}{|c|c|c|c|c|c|c|c|}
\hline \multirow[b]{4}{*}{ Model } & \multirow{2}{*}{\multicolumn{5}{|c|}{ Soft state $\quad N u S T A R$}} & \multirow{2}{*}{\multicolumn{2}{|c|}{ Hard state }} \\
\hline & & & & & & & \\
\hline & \multirow{2}{*}{$\begin{array}{l}2015 \text { March } 31 \\
\text { DBB-BB-NTH-RR }\end{array}$} & \multirow{2}{*}{$\begin{array}{l}2013 \text { August } 23 \\
\text { DBB-BB-NTH-RR }\end{array}$} & \multirow{2}{*}{$\begin{array}{l}\text { Soft state } \\
2013 \text { August } 13 \\
\text { DBB-BB-NTH-RR }\end{array}$} & \multirow{2}{*}{$\begin{array}{l}2013 \text { August } 09 \\
\text { DBB-BB-NTH-RR }\end{array}$} & \multirow{2}{*}{$\begin{array}{l}2013 \text { August } 08 \\
\text { DBB-BB-NTH-RR }\end{array}$} & \multirow{2}{*}{$\begin{array}{l}2014 \text { June } 18 \\
\text { DBB-NTH }\end{array}$} & \multirow{2}{*}{$\begin{array}{l}2013 \text { July } 31 \\
\text { DBB-NTH }\end{array}$} \\
\hline & & & & & & & \\
\hline$k T_{\mathrm{DBB}}$ & $1.5_{-0.4}^{+0.1}$ & $1.2 \pm 0.2$ & $1.5_{-0.3}^{+0.1}$ & $1.5_{-0.4}^{+0.2}$ & $1.6_{-0.4}^{+0.2}$ & - & - \\
\hline$N_{\mathrm{DBB}}$ & $7_{-1}^{+4}$ & $15 \pm 10$ & $6_{-1}^{+6.3}$ & $6_{-2}^{+15}$ & $3_{-1}^{+17}$ & - & - \\
\hline$k T_{\mathrm{BB}}$ & $3.0 \pm 0.4$ & $2.3 \pm 0.2$ & $2.7 \pm 0.3$ & $2.5 \pm 0.4$ & $2.5 \pm 0.3$ & - & - \\
\hline$r_{\mathrm{BB}}$ & $0.11_{-0.02}^{+0.12}$ & $0.6 \pm 0.2$ & $0.2_{-0.1}^{+0.3}$ & $0.3_{-0.2}^{+0.4}$ & $0.3 \pm 0.2$ & - & - \\
\hline$\Gamma$ & $1.1^{-0.02}$ & 1.1 & 1.1 & 1.1 & 1.1 & $1.94 \pm 0.04$ & $1.93 \pm 0.05$ \\
\hline$k T_{\mathrm{e}}$ & 4.5 & 4.5 & 4.5 & 4.5 & 4.5 & $>90$ & $>90$ \\
\hline$N_{\text {nth }}$ & $<0.01$ & $0.009 \pm 0.003$ & $<0.01$ & $0.010 \pm 0.005$ & $<0.01$ & $2.6 \pm 0.2$ & $3.3 \pm 0.02$ \\
\hline$N_{\text {ref }}$ & $33_{-9}^{+15}$ & $42_{-21}^{+33}$ & $19_{-10}^{+20}$ & $17_{-4}^{+10}$ & $15 \pm 15$ & & \\
\hline$c_{\mathrm{NuB}}$ & $1.03 \pm 0.01$ & $1.03 \pm 0.01$ & $1.02 \pm 0.01$ & $1.04 \pm 0.02$ & $1.03 \pm 0.02$ & $1.09 \pm 0.02$ & $1.02 \pm 0.02$ \\
\hline$\chi^{2} /$ dof & $542.1 / 634$ & $735.3 / 700$ & $560.4 / 519$ & $496.8 / 514$ & $578.8 / 519$ & $909.1 / 837$ & $839.4 / 772$ \\
\hline
\end{tabular}

\subsection{Soft-state observations}

All soft-state observations of AX J1745.6-2901 show clear signs of ionized absorption lines in the XMM-Newton and NuSTAR spectra, signatures of a highly ionized $\left(\log \left(\xi_{\mathrm{IA}}\right) \sim 4.1\right)$ and high column density $\left(\log \left(N_{\mathrm{H}_{\mathrm{IA}}}\right) \sim 23.5-24\right)$ plasma. We note that these values are within the range of typical ionization states and column densities observed in NS and BH LMXB (King et al. 2013; Ponti et al. 2016a; Díaz Trigo \& Boirin 2016). Because of the limited energy resolution, we can only place weak constraints on the bulk outflow velocity of this plasma $\left(v_{\text {out }} \leq 2000 \mathrm{~km} \mathrm{~s}^{-1}\right)$. The ionized plasma is consistent with being constant within all soft-state observations.

During the soft state, once the neutral and ionized absorptions are reproduced, broad positive residuals appear in the $\sim 5-7 \mathrm{keV}$ band. We explored whether such excess could be the manifestation of the emission component of a P-Cygni profile. Nevertheless, the broadness of this feature is inconsistent with the outflow velocities typically observed in winds in NS and constrained in AX J1745.62901. Alternatively, we explored whether such feature might be reproduced by a broad $\mathrm{Fe} \mathrm{K} \alpha$ emission line, reflected off the accretion disc. The introduction of a disc-line profile provides an acceptable fit, with a line equivalent width $\mathrm{EW} \sim 120-200 \mathrm{eV}$, consistent with disc reflection (Matt et al. 1993). The result for the best-fitting disc inclination is high, roughly consistent with the eclipsing behaviour of the source, and the disc emissivity index is scaling with radius as $r^{-2.4 \pm 0.1}$, a value consistent with illumination of a flared disc by a central source. We note that for all combinations of continuum emission models the substitution of the disc-line component with a self-consistent ionized reflection spectrum provides a significant improvement of the fit. Although we cannot rule out alternative hypotheses (e.g. more complex absorption, etc.), this strengthens the suggestion that the positive excess in the $5-7 \mathrm{keV}$ band has an origin as reprocessing from the accretion disc.

The soft X-ray spectrum (within the XMM-Newton band) of AX J1745.6-2901 is dominated by a prominent thermal component. The three emission component (DBB-BB-NTH-RR) model provides a superior fit and an excellent description of all soft state spectra. In this model, the continuum is produced by a prominent disc blackbody component with a temperature of $k T_{\mathrm{DBB}}=1.0-1.2 \mathrm{keV}$, dominating the emission below $\sim 5 \mathrm{keV}$. Such a range of temperatures is theoretically expected and typically observed in BH and NS in the soft state. Under the assumption that AXJ is located within the GC and that its disc is highly inclined $\left(i \sim 80^{\circ}\right.$, indeed it shows eclipses) and some correction factors (see Kubota et al. 1998), the best-fitting inner disc radius results to be $r_{\mathrm{DBB}} \sim 12-16 \mathrm{~km}\left(\sim 7 r_{g}\right)$. As expected, this value is comparable (however larger) than the typical NS radius. In the best-fitting model (DBB-BB-NTH-RR), the second emission component, required to reproduce the hard X-ray emission measured by $N U S T A R$, is associated with blackbody emission, possibly connected to the boundary layer at the NS surface. This component dominates the source emission in the $\sim 8-20 \mathrm{keV}$ band. Its temperature ranges within $k T_{\mathrm{BB}} \sim 2.2-3.0 \mathrm{keV}$ with an associated very small emitting radius of $r_{\mathrm{BB}} \sim 0.5-0.8 \mathrm{~km}$. In theory, this radiation might be associated with emission from small and hot patches or an equatorial bundle on the NS surface (e.g. where the accretion column impacts the NS surface). The emission above $\sim 20 \mathrm{keV}$ can be fit by the addition of a faint Comptonization component. In this three component model, the Comptonization emission is very weak, preventing a detailed characterization of its parameters. The best-fitting asymptotic photon index and electron temperature are $\Gamma=1.1_{-0.2}^{+1.1}$ and $k T_{\mathrm{e}}=4.6_{-2.4}^{+10.3} \mathrm{keV}$, respectively. These values carry large uncertainties; however, they indicate a significant optical thickness of this medium with a scattering optical depths $(\tau)$ of $\tau$ $\geq 3$ (assuming the relation $\Gamma=\left[9 / 4+\frac{1}{\left(k T_{\mathrm{e}} / m_{\mathrm{e}} c^{2}\right) \tau(1+\tau / 3)}\right]^{1 / 2}-1 / 2$; Sunyaev \& Titarchuk 1980). Finally, the addition of a relativistic ionized reflection model also improves the fit. We observed that in all cases, the combination of disc blackbody plus blackbody emission carry most ( $\sim 70-75$ per cent) of the flux within the $1-200 \mathrm{keV}$ band and the ratio of blackbody to disc blackbody radiation is $\sim 0.4$ 0.8 . On the other hand, the Comptonization component carries only $\sim 10$ per cent of the $1-200 \mathrm{keV}$ emission, while a significant contribution $(\sim 20-30$ percent $)$ is provided by the relativistic ionized reflection.

We note that in the three component model, the bulk of the $\sim 8-20 \mathrm{keV}$ emission is produced by the blackbody radiation and the Comptonized component is very faint, and relegated to higher energies. Both the spectral parameters and the relative contributions of the emission components are consistent with those observed in a very similar system, $4 \mathrm{U} 1608-52$, during the soft state and using the same modelling (Armas Padilla et al. 2017). This system is seen through an $N_{\mathrm{H}}$ 20-30 times lower than that of AX J1745.6-2901, which enables to study the softest spectral region $(<3 \mathrm{keV})$. We also note that the low Comptonization fractions inferred from the three component model are systematically seen in BH soft states (e.g. 
Dunn et al. 2011 ; Muñoz-Darias et al. 2013). This behaviour seems reasonable given that both $\mathrm{BH}$ and NS systems reach similarly low fast variability levels during the soft state, which is likely to be produced in the Comptonization component (e.g. Muñoz-Darias et al. 2014; see also Lin et al. 2007, 2009).

Alternatively, the soft-state spectra are reasonably well reproduced by a two component model (e.g. disc blackbody emission plus Comptonization). In this case, the Comptonized component would be more prominent, producing most of the emission above $8 \mathrm{keV}$. If so, a high-energy cut-off must occur within the X-ray band, implying that the Comptonized emission is produced by a population of low-temperature electrons $\left(k T_{\mathrm{e}} \sim 3-4 \mathrm{keV}\right)$.

We stress that this electron temperature is one to two orders of magnitude smaller than what is typically observed in $\mathrm{BH}$ binaries (both soft and hard states) and in NS during the hard state. In this scenario, the best-fitting photon index is $\Gamma=1.7-2.2$, therefore consistent with the values typically observed in accreting $\mathrm{BH}$ and NS. The observed photon index and electron temperature imply a very high optical depth of this plasma $(\tau \sim 8-12)$. This means that, even when the high-energy radiation is fitted with a (non-thermal) Comptonization model, the Comptonized radiation goes into the limit of becoming nearly-thermal blackbody radiation, right where the Comptonization radiation and blackbody blur.

\subsection{Hard-state observations}

During the hard state, AX J1745.6-2901's X-ray emission is dominated by a power law with photon index $\Gamma \sim 1.8-2.0$, showing no evidence for a high-energy cut-off $\left(k T_{\mathrm{e}}>70-100 \mathrm{keV}\right)$. This implies a small optical depth of the Comptonizing plasma $(\tau<1.6)$. Would the high-energy cut-off be located at $k T_{\mathrm{e}}=300$ or $k T_{\mathrm{e}}=800 \mathrm{keV}$, then the Comptonizing plasma optical depth would be $\tau \sim 0.4$ 0.15 , resulting into a very optically thin layer. The large lower limit to the energy of the cut-off appears rather high compared to what is typically observed in accreting NS (Burke et al. 2017) and this might be related to inclination effects (Makishima et al. 2008).

We also confirm previous results observing the disappearance of the ionized absorption plasma in the hard state. As already discussed in previous works, it is excluded that the absorption disappears because of overionization, during the hard state (Ponti et al. 2015a). To understand the origin of this variation, we constructed here detailed and accurate SED to use as input for investigating the photoionization stability of the absorbing plasma. We will show in a companion paper that, whenever the ionized plasma observed during the soft state is illuminated by a hard-state SED, it becomes unstable. Therefore, it has to change its physical conditions (Bianchi et al. 2017).

Tight upper limits are observed also on the presence of broad emission lines $(\mathrm{EW}<30 \mathrm{eV})$, during the hard state. This could be the consequence of a major variation in the accretion flow, with the optically thick layer accretion disc that is ubiquitous during the soft state becoming optically thin (therefore producing no reflection component) inside a (rather large) truncation radius, during the hard state (Done et al. 2007; Plant et al. 2014, 2015; De Marco et al. 2015; De Marco and Ponti 2016).

\section{CONCLUSIONS}

We presented 11 new XMM-Newton observations as well as 15 new NUSTAR data sets that caught AX J1745.6-2901 in outburst, therefore building a large data base (of almost 40 observations) of good resolution X-ray spectra of a high inclination NS X-ray binary accreting at intermediate rates (i.e. atoll regime). (i) We built accurate X-ray SEDs, representative of each state, starting from the best-fitting models best reproducing the $X M M$ Newton and NuSTAR spectra. We also reported radio (GMRT) and optical (GROND) upper limits that are consistent with the known radio and optical to $\mathrm{X}$-ray relations.

(ii) All soft-state observations are well described by a three component model. The best fit is provided by a disc blackbody component with $k T_{\mathrm{DBB}} \sim 1.1-1.2 \mathrm{keV}$ an inner disc radius $r_{\mathrm{DBB}} \sim 12-16$ $\mathrm{km} \sim 7 r_{g}$, plus a hot $k T_{\mathrm{BB}} \sim 2.2-3.0 \mathrm{keV}$ blackbody component with a small emitting radius $r_{\mathrm{BB}} \sim 0.5-0.8 \mathrm{~km}$, possibly produced by the boundary layer at the NS surface, plus a faint Comptonization. Additionally, neutral plus ionized absorption and relativistic ionized reflection components are required by the data.

(iii) All hard-state observations are dominated by hard X-ray radiation, well reproduced by a rather flat power law emission $(\Gamma \sim 1.8-2.0)$. No significant curvature is detected in the $X M M$ Newton + NuSTAR band, indicating no requirement for a highenergy cut-off up to $\sim 70-140 \mathrm{keV}$. This implies a small optical depth of the Comptonizing plasma $(\tau<1.6)$.

(iv) We confirm, tripling the number of X-ray observations, the ubiquitous presence of $\mathrm{Fe} \mathrm{K}$ absorption lines during the soft state $\log \left(\xi_{\mathrm{IA}}\right) \sim 3.7-4.3, \log \left(N_{\mathrm{H}_{\mathrm{IA}}}\right) \sim 23.4-23.5, v_{\text {out }}<2000 \mathrm{~km} \mathrm{~s}^{-1}$ and $v_{\text {turb }} \sim 500-700 \mathrm{~km} \mathrm{~s}^{-1}$. The plasma physical parameters remain roughly constant during all the soft-state observations, while the ionized absorption features are significantly weakening during the hard state, as observed in archival data (Ponti et al. 2015a). We will investigate the dependence of the plasma properties on the source SED (therefore on its photo-ionization stability) in a companion paper (Bianchi et al. 2017).

(v) During all soft-state observations positive residuals remain in the $\sim 6-8 \mathrm{keV}$ band. Such emission can be well reproduced by a reflection component with an Fe $\mathrm{K} \alpha$ line with $\mathrm{EW} \sim 120-200 \mathrm{eV}$. The best-fitting parameters of the broad emission line indicate a rather standard disc emissivity $\left(r^{-2.4 \pm 0.1}\right)$ from a highly inclined accretion disc $\left(\alpha=70_{-15}^{+7}\right)$.

The disc line is not observed during the hard state, in line with the idea that the disc might be truncated, during the hard state (e.g. Plant et al. 2014, 2015; De Marco et al. 2015; De Marco \& Ponti 2016).

(vi) Although the ionized absorption is highly variable observations in the soft state and in the hard state, a constant column density of neutral absorption can fit all XMM-Newton spectra and all NUSTAR spectra of the persistent (out of dip) emission of AX J1745.6-2901. This would be expected if the majority of the neutral absorption is due to material in the interstellar medium (Jin et al. 2017).

\section{ACKNOWLEDGEMENTS}

The authors wish to thank Jan-Uwe Ness, Karl Foster, Ignacio de la Calle and the rest of the XMM-Newton and NUSTAR scheduling teams for the support that made the coordinated observations possible. We would like to thank the referee for the helpful comments and careful reading of the paper. The GC XMM-Newton monitoring project is supported by the Bundesministerium für Wirtschaft und Technologie/Deutsches Zentrum für Luft- und Raumfahrt (BMWI/DLR, FKZ 50 OR 1408 and FKZ 50 OR 1408) and the Max Planck Society. SB acknowledges financial support from the Italian Space Agency under grant ASI-INAF I/037/12/0. TMD acknowledges support via a Ramón y Cajal Fellowship (RYC-2015-18148). BDM acknowledges support from the European Union's Horizon 2020 research and innovation programme and the Polish National 
Science Centre grant Polonez 2016/21/P/ST9/04025. These results are based on observations obtained with XMM-Newton, an ESA science mission with instruments and contributions directly funded by ESA Member States and NASA. This work made use of data from the NUSTAR mission, a project led by the California Institute of Technology, managed by the Jet Propulsion Laboratory and funded by the National Aeronautics and Space Administration. We thank the NUSTAR Operations, Software and Calibration teams for support with the execution and analysis of these observations. This research has made use of the NUSTARDAS jointly developed by the ASI Science Data Centre (ASDC, Italy) and the California Institute of Technology (USA). We thank the staff of the GMRT who have made these observations possible. The GMRT is run by the National Centre for Radio Astrophysics of the Tata Institute of Fundamental Research. Part of the funding for GROND (both hardware as well as personnel) was generously granted from the Leibniz-Prize to Prof. G. Hasinger (DFG grant HA 1850/28-1).

\section{REFERENCES}

Armas Padilla M., Ueda Y., Hori T., Shidatsu M., Muñoz-Darias T., 2017, MNRAS, 467, 290

Arnaud K. A., 1996, in Jacoby G. H., Barnes J., eds., ASP Conf. Ser. Vol. 101, Astronomical Data Analysis Software and Systems V. Astron. Soc. Pac., San Francisco, p. 17

Ballantyne D. R., 2004, MNRAS, 351, 57

Barret D., 2001, Adv. Space Res., 28, 307

Barret D., Olive J.-F., 2002, ApJ, 576, 391

Barret D., Olive J. F., Boirin L., Done C., Skinner G. K., Grindlay J. E., 2000, ApJ, 533, 329

Belloni T., Psaltis D., van der Klis M., 2002, ApJ, 572, 392

Belloni T. M., Motta S. E., Muñoz-Darias T., 2011, Bull. Astron. Soc. India, 39, 409

Bianchi S., Ponti G., Muñoz-Darias T., Petrucci P.-O., 2017, MNRAS, preprint (arXiv: 1709.00860)

Bland-Hawthorn J., Gerhard O., 2016, ARA\&A, 54, 529

Brandt W. N., Schulz N. S., 2002, ApJ, 544, L123

Burke M. J., Gilfanov M., Sunyaev R., 2017, MNRAS, 466, 194

Church M. J., Balucinska-Church M., 1995, A\&A, 300, 441

Church M. J., Balucińska-Church M., 2001, A\&A, 369, 915

Church M. J., Bałucińska-Church M., 2004, MNRAS, 348, 955

Corral-Santana J. M., Casares J., Muñoz-Darias T., Bauer F. E., MartnezPais I. G., Russell D. M., 2016, A\&A, 587, A61

Coti Zelati F. et al., 2015, MNRAS, 449, 2685

De Marco B., Ponti G., 2016, ApJ, 826, 70

De Marco B., Ponti G., Muñoz-Darias T., Nandra K., 2015, ApJ, 814, 50

Degenaar N., Wijnands R., 2009, A\&A, 495, 547

Degenaar N., Miller J. M., Kennea J., Gehrels N., Reynolds M. T., Wijnands R., 2013, ApJ, 769, 155

Degenaar N., Wijnands R., Reynolds M. T., Miller J. M., Kennea J., Gehrels N., 2014, in Sjouwerman L. O., Lang C. C., Ott J., eds, Proc. IAU Symp., 303, The Galactic Center: Feeding and Feedback in a Normal Galactic Nucleus, p. 315

Degenaar N., Reynolds M. T., Wijnands R., Miller J. M., Kennea J. A., Ponti G., Haggard D., Gehrels N., 2016, Astron. Telegram, 9196

Di Salvo T. et al., 2000, ApJ, 544, L119

Díaz Trigo M., Boirin L., 2016, Astron. Nachr., 337, 368

Díaz Trigo M., Parmar A. N., Boirin L., Méndez M., Kaastra J. S., 2006, A\&A, 445, 179

Done C., Díaz Trigo M., 2010, MNRAS, 407, 2287

Done C., Gierliński M., 2006, MNRAS, 367, 659

Done C., Gierliński M., Kubota A., 2007, A\&ARv, 15, 1

Dunn R. J. H., Fender R. P., Körding E. G., Belloni T., Cabanac C., 2010, MNRAS, 403, 61

Dunn R. J. H., Fender R. P., Körding E. G., Belloni T., Merloni A., 2011, MNRAS, 411, 337
Fabian A. C., Ross R. R., 2010, SSRv, 157, 167

Fabian A. C., Rees M. J., Stella L., White N. E., 1989, MNRAS, 238, 729

Fabian A. C., Iwasawa K., Reynolds C. S., Young A. J., 2000, PASP, 112, 1145

Falcke H., Körding E., Markoff S., 2004, A\&A, 414, 895

Fender R., Muñoz-Darias T., 2016, Lecture Notes in Physics, Vol. 905. Springer Verlag, Berlin, p. 65

Fender R. P., Belloni T. M., Gallo E., 2004, MNRAS, 355, 1105

Ferland G. J. et al., 2013, Rev. Mex. Astron. Astrofis., 49, 137

Frank J., King A. R., Lasota J.-P., 1987, A\&A, 178, 137

Fritz T. K. et al., 2011, ApJ, 737, 73

Genzel R. et al., 2010, MNRAS, 407, 2091

Gierliński M., Done C., 2002, MNRAS, 337, 1373

Gillessen S. et al., 2016, ApJ, 837, 30

Greiner J. et al., 2008, PASP, 120, 405

Harrison F. A. et al., 2013, ApJ, 770, 103

Hasinger G., van der Klis M., 1989, A\&A, 225, 79

Hong J. et al., 2015, ApJ, 825, 132

Hyodo Y., Ueda Y., Yuasa T., Maeda Y., Makishima K., Koyama K., 2009, PASJ, 61, 99

Iaria R., Spanò M., Di Salvo T., Robba N. R., Burderi L., Fender R., van der Klis M., Frontera F., 2005, ApJ, 619, 503

Jansen F. et al., 2001, A\&A, 365, L1

Jin C., Ponti G., Haberl F., Smith R., 2017, MNRAS, 468, 2532

Kaspi V. M. et al., 2014, ApJ, 786, 84

King A. L. et al., 2013, ApJ, 762, 103

King A. L., Miller J. M., Raymond J., Reynolds M. T., Morningstar W., 2015, ApJ, 813, L37

Kolehmainen M., Done C., Díaz Trigo M., 2014, MNRAS, 437, 316

Krühler T. et al., 2008, ApJ, 685, 376

Kubota A., Tanaka Y., Makishima K., Ueda Y, Dotani T., Inoue H., Yamaoka K., 1998, PASJ, 50, 667

Laor A., 1991, ApJ, 376, 90

Lee J. C., Reynolds C. S., Remillard R., Schulz N. S., Blackman E. G., Fabian A. C., 2002, ApJ, 567, 1102

Lewin W. H. G., van Paradijs J., Taam R. E., 1993, SSRv, 62, 223

Lin D., Remillard R. A., Homan J., 2007, ApJ, 667, 1073

Lin D., Remillard R. A., Homan J., 2009, ApJ, 696, 1257

Liu Q. Z., van Paradijs J., van den Heuvel E. P. J., 2000, A\&AS, 147, 25

Liu Q. Z., van Paradijs J., van den Heuvel E. P. J., 2001, A\&A, 368, 1021

Madsen K. K. et al., 2015, ApJS, 220, 8

Maeda Y., Koyama K., Sakano M., Takeshima T., Yamauchi S., 1996, PASJ, 48,417

Makishima K. et al., 1986, ApJ, 308, 635

Makishima K. et al., 2008, PASJ, 60, 585

Matt G., Fabian A. C., Ross R. R., 1993, MNRAS, 262, 179

Miller J. M., Homan J., Steeghs D., Rupen M., Hunstead R. W., Wijnands R., Charles P. A., Fabian A. C., 2006a, ApJ, 653, 525

Miller J. M., Raymond J., Fabian A., Steeghs D., Homan J., Reynolds C., van der Klis M., Wijnands R., 2006b, Nature, 441, 953

Miller J. M. et al., 2006c, ApJ, 646, 394

Miller L., Turner T. J., Reeves J. N., George I. M., Kraemer S. B., Wingert B., 2007, A\&A, 463, 131

Miller J. M., Fabian A. C., Kaastra J., Kallman T., King A. L., Proga D., Raymond J., Reynolds C. S., 2015, ApJ, 814, 87

Mitsuda K. et al., 1984, PASJ, 36, 741

Mitsuda K., Inoue H., Nakamura N., Tanaka Y., 1989, PASJ, 41, 97

Mori K. et al., 2013, ApJ, 770, L23

Mori K. et al., 2015, ApJ, 814, 94

Motta S. E., Rouco-Escorial A., Kuulkers E., Muñoz-Darias T., Sanna A., 2017, MNRAS, 468, 2311

Muñoz-Darias T., Coriat M., Plant D. S., Ponti G., Fender R. P., Dunn R. J. H., 2013, MNRAS, 432, 1330

Muñoz-Darias T., Fender R. P., Motta S. E., Belloni T. M., 2014, MNRAS, 443,3270

Muñoz-Darias T. et al., 2016, Nature, 534, 75

Muñoz-Darias T. et al., 2017, MNRAS, 465, L124

Nandra K., O’Neill P. M., George I. M., Reeves J. N., 2007, MNRAS, 382, 194 
Oosterbroek T., Barret D., Guainazzi M., Ford E. C., 2001, A\&A, 366, 138 Parmar A. N., Oosterbroek T., Boirin L., Lumb D., 2002, A\&A, 386, 910

Petrucci P.-O., Cabanac C., Corbel S., Koerding E., Fender R., 2014, A\&A, 564, A37

Plant D. S., Fender R. P., Ponti G., Muñoz-Darias T., Coriat M., 2014, MNRAS, 442, 1767

Plant D. S., Fender R. P., Ponti G., Muñoz-Darias T., Coriat M., 2015, A\&A, 573, A120

Ponti G., Miniutti G., Cappi M., Maraschi L., Fabian A. C., Iwasawa K., 2006, MNRAS, 368, 903

Ponti G. et al., 2010, MNRAS, 406, 2591

Ponti G., Fender R. P., Begelman M. C., Dunn R. J. H., Neilsen J., Coriat M., 2012, MNRAS, 422, L11

Ponti G., Muñoz-Darias T., Fender R. P., 2014, MNRAS, 444, 1829

Ponti G. et al., 2015a, MNRAS, 446, 1536

Ponti G. et al., 2015b, MNRAS, 454, 1525

Ponti G. et al., 2015c, MNRAS, 453, 172

Ponti G., Bianchi S., Muñoz-Darias T., De K., Fender R., Merloni A, 2016a, Astron. Nachr., 337, 512

Ponti G. et al., 2016b, MNRAS, 461, 2688

Ponti G., De K., Muñoz-Darias T., Stella L., Nandra K., 2017, MNRAS, 464,840

Ponti G. et al., 2017, MNRAS, 468, 2447

Predehl P., Schmitt J. H. M. M., 1995, A\&A, 293, 889

Rea N. et al., 2013, ApJ, 775, L34

Reig P., van Straaten S., van der Klis M., 2004, ApJ, 602, 918

Reis R. C., Fabian A. C., Ross R. R., Miniutti G., Miller J. M., Reynolds C., 2008, MNRAS, 387, 1489

Reis R. C., Fabian A. C., Miller J. M., 2010, MNRAS, 402, 836

Reynolds C. S., Nowak M. A., 2003, Phys. Rep., 377, 389

Schlafly E. F., Finkbeiner D. P., 2011, ApJ, 737, 103

Shidatsu M. et al., 2011, PASJ, 63, S785

Skrutskie M. F. et al., 2006, AJ, 131, 1163

Steiner J. F., Narayan R., McClintock J. E., Ebisawa K., 2009, PASP, 121, 1279

Strüder L. et al., 2001, A\&A, 365, L18

Sunyaev R. A., Titarchuk L. G., 1980, A\&A, 86, 121

Swarup G., Ananthakrishnan S., Subrahmanya C. R., Rao A. P., Kulkarni V. K., Kapahi V. K., 1997, in Jackson D., ed., High-Sensitivity Radio Astronomy. Cambridge Univ. Press, Cambridge, p. 217

Tetarenko B. E., Sivakoff G. R., Heinke C. O., Gladstone J. C., 2016, ApJS, 222,15

Tomsick J. A., Yamaoka K., Corbel S., Kaaret P., Kalemci E., Migliari S., 2009, ApJ, 707, L87

Turner M. J. L. et al., 2001, A\&A, 365, L27

Ueda Y., Murakami H., Yamaoka K., Dotani T., Ebisawa K., 2004, ApJ, 609,325

van der Klis M., 2006, Compact Stellar X-ray Sources, 39

Verner D. A., Ferland G. J., Korista K. T., Yakovlev D. G., 1996, ApJ, 465 , 487

Voges W. et al., 1999, A\&A, 349, 389

Wang Q. D., Gotthelf E. V., Lang C. C., 2002, Nature, 415, 148

White N. E., Stella L., Parmar A. N., 1988, ApJ, 324, 363

Wijnands R., van der Klis M., 1999, ApJ, 514, 939

Wik D. R. et al., 2014, ApJ, 792, 48

Wilms J., Allen A., McCray R., 2000, ApJ, 542, 914

Yamada S. et al., 2009, ApJ, 707, L109

Zdziarski A. A., Johnson W. N., Magdziarz P., 1996, MNRAS, 283, 193

Zhang S. et al., 2015, ApJ, 815, 132

Życki P. T., Done C., Smith D. A., 1999, MNRAS, 309, 561

\section{APPENDIX A: FURTHER DETAILS ON NUSTAR DATA REDUCTION}

\section{A1 Bright transients}

The immense improvement in the NUSTAR PSF, compared to previous hard $\mathrm{X}$-ray telescopes, allows us to accurately study the hard
X-ray emission from relatively faint sources like AX J1745.6-2901 in extremely crowded fields such as the GC (Hong et al. 2015; Mori et al. 2015). The four panels in Fig. A1 show the NuSTAR RGB image of the regions around AX J1745.6-2901. The top left-hand panel shows the X-ray emission on 2012-07-20, when no bright $\mathrm{X}$-ray transient was observed. Bright and diffuse X-ray emission is permeating the $\mathrm{GC}$ regions, producing a highly spatially variable background (see also Wang, Gotthelf \& Lang 2002; Mori et al. 2015; Ponti et al. 2015c; Fig. A1). The right-hand panel shows the X-ray emission on 2013-04-27, just after the start of the long outburst of SGR J1745-2900, the magnetar that is located at $\sim 1.45$ arcmin from AX J1745.6-2901 and only $\sim 2.4$ arcsec from Sgr A* (Mori et al. 2013; Rea et al. 2013; Kaspi et al. 2014). We note that despite SGR J1745-2900 is lying outside of the extraction circle of AX J1745.6-2901, a fraction of SGR J1745-2900's photons enter into AX J1745.6-2901's extraction region, polluting the spectra of AX J1745.6-2901. SGR J1745-2900's spectrum is very soft, it is best fit with $k T \sim 0.7-0.9 \mathrm{keV}$, an absorbed $1-10 \mathrm{keV}$ flux of $F_{1-10} \sim(2-20) \times 10^{-12} \mathrm{erg} \mathrm{cm}^{-2} \mathrm{~s}^{-1}$, absorbed by a column density of neutral material of $N_{\mathrm{H}} \sim 1.5-2 \times 10^{23} \mathrm{~cm}^{-2}$, exponentially decreasing over the period 2013-2014 (Mori et al. 2013; Rea et al. 2013; Coti Zelati et al. 2015; Kaspi et al. 2014). The bottom panels of Fig. A1 show that the emission of AX J1745.6-2901 dominates over that of SGR J1745-2900 when it was in outburst (see the bottom left-hand and right-hand panels of Fig. A1).

We note that during the observation performed on 2016 February 18 (obsid: 90101022002), Swift J174540.7-290015, the accreting binary located at only $\sim 1.5$ arcmin from AX J1745.6-2901, was in

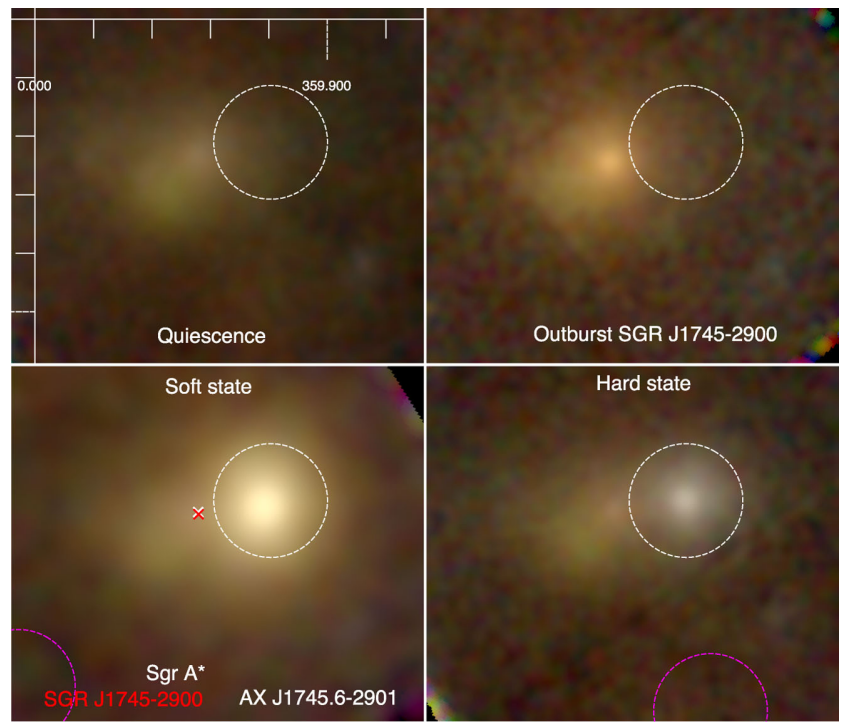

Figure A1. NUSTAR exposure corrected RGB images of AX J1745.6-2901 (red, green and blue show the 3-6, 6-10 and 10-60 keV band, respectively). The white dashed circle shows the region used to extract the spectrum of AX J1745.6-2901. The white and red crosses show the position of Sgr A ${ }^{\star}$ and SGR J1745-2900, respectively. The top left-hand panel shows the GC on 2012-07-20, when no bright X-ray transient was observed and AX J1745.62901 was in quiescence. The scale is in Galactic coordinates. The top righthand shows the GC X-ray emission on 2013-04-27, just before the outburst of AX J1745.6-2901 and around the peak of the outburst of SGR J17452900. The bottom left-hand and right-hand panels show the X-ray emission on 2015-02-25 and 2014-09-27, while AX J1745.6-2901 was in the soft and hard states, respectively. The magenta dashed circle shows the region used to extract the local background. The same logarithmic colour scale has been applied to all maps. 


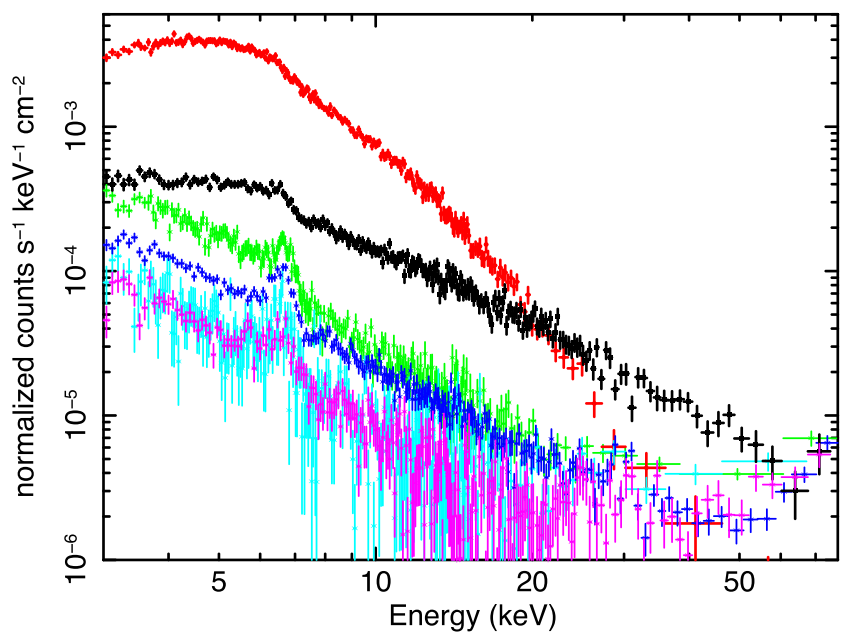

Figure A2. NUSTAR spectra of AX J1745.6-2901 and of the background. The red dots and black squares show the spectra of AX J1745.6-2901 during the soft (2015 February 25) and hard states (2014 September 27), respectively. The blue asterisks show the background spectrum, from the same region used to extract the photons of AX J1745.6-2901, when the source was quiescent and no X-ray transient was detected (2012 July 20). The green asterisks show the background from the same region, when SGR J1745-2900 was in outburst (2013 April 27). The cyan and magenta spectra show the local background spectra extracted from the magenta regions shown in Fig. A1, during the soft and hard states, respectively.

outburst and brighter than AX J1745.6-2901, therefore hampering a proper study of the spectrum of AX J1745.6-2901 (Degenaar et al. 2016; Ponti et al. 2016). For this reason, we excluded this observation from further analysis.

\section{A2 Background}

To evaluate the importance on our results of the bright and highly spatially variable background emission, we extracted the background photons from several regions.

We initially selected the background during the longest NuSTAR exposure (accumulated in 2012 July 20; 156.8 ks), when AX J1745.6-2901 was in quiescence and no bright transient within a few arcmin from $\mathrm{Sgr} \mathrm{A}^{\star}$ was active (see Fig. A1). We extracted the background from the same region used for AX J1745.6-2901's source emission (see Section 2.2; a circular region with 70arcsec radius centred on AX J1745.6-2901). The blue asterisks in Fig. A2 show such background emission. We will refer to this background as BackQuie. We note that the BackQuie background dominates over the spectrum of AX J1745.6-2901 at energies above $\sim 30-40 \mathrm{keV}$ during the soft (red) state and above $\sim 60-70 \mathrm{keV}$ in the hard (black) state. Therefore, to avoid contamination from the background emission, we discarded the source photons above 40 and $70 \mathrm{keV}$ during the soft and hard states, respectively.

To estimate the maximum contamination of SGR J1745-2900 to the spectrum of AX J1745.6-2901, we extracted a background spectrum from the same region used to extract AX J1745.6-2901's photons, from an observation obtained on 2013 Apil 27, when AX J1745.6-2901 was quiescent and SGR J1745-2900 was at the peak of the X-ray outburst (Fig. A1). We will refer to this background spectrum (green asterisks in Fig. A2) as BackSGR. To extract a quantitative measure of the emission induced by SGR J1745-2900 to the spectrum of AX J1745.6-2901, we then fit the BackSGR spectrum, using BackQuie as background, with an absorbed blackbody

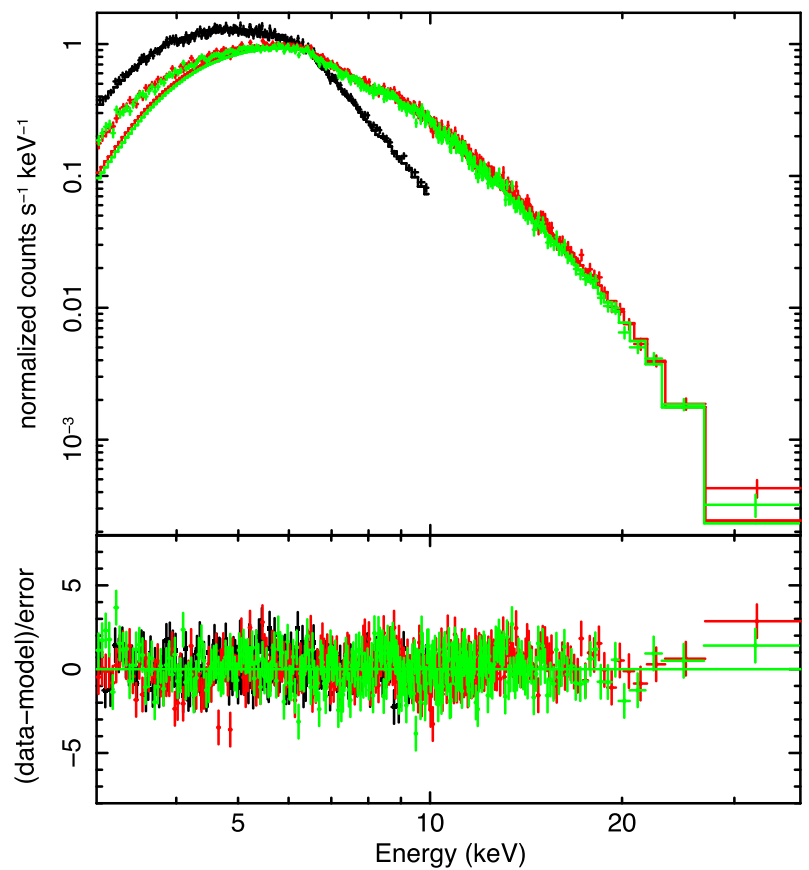

Figure A3. Top panel:The black, red and green data show the $X M M$ Newton, NuSTAR FPMA and FPMB spectra obtained during the soft state simultaneous observation with the longest exposure (Tables A1 and A2). The XMM-Newton and NUSTAR spectra are fitted over the 3-10 and 5-40 keV energy band. Clear deviations are observed at low energy when such model (best fitting the XMM-Newton data) is extrapolated in the $3-5 \mathrm{keV}$ range. Bottom panel: Residuals after fitting the spectra with model DBB-BB-RR, allowing the column density of neutral material derived from the NUSTAR spectra to be different from the XMM-Newtonone (modified absorption).

model (with $N_{\mathrm{H}}=1.6 \times 10^{23} \mathrm{~cm}^{-2}$; Ponti et al. 2017). The bestfitting values are $k T_{\mathrm{BB}}=0.73 \mathrm{keV}$ and $r_{\mathrm{BB}}=2.5 \mathrm{~km}$.

We also extracted local background spectra from various regions around AX J1745.6-2901. The cyan and magenta spectra in Fig. A2 show the local background from the observation accumulated on 2015 February 25 and 2014 September 27, when AX J1745.6-2901 was in the soft and hard states, respectively (see the magenta regions shown in Fig. A1). We chose these local background extraction regions by requiring them to be: (i) located away from AX J1745.62901 and $\mathrm{Sgr} \mathrm{A}^{\star}$; (ii) to be within the field of view; (iii) to avoid $\mathrm{X}$-ray transients as well as and (iv) stray light and ghost rays.

The comparison between the local and quiescent (BackQuie) backgrounds indicates that the latter are dominated by the diffuse GC emission, with only a small contribution from detector and particle background up to 30-40 keV (see Mori et al. 2015; Zhang et al. 2015 for more details). To correctly subtract the GC diffuse emission, we therefore chose to use BackQuie as background for the spectral analysis.

\section{A3 NuSTAR versus XMM-Newton low energy small mismatch}

\section{A3.1 Soft state}

The black, red and green data in Fig. A3 show the XMM-Newton, NuSTAR FPMA and FPMB spectra, respectively, obtained during the soft-state observation with the longest simultaneous exposure (Tables A1 and A2). We fit the XMM-Newton and NuSTAR data with model DBB-BB-DL (see Section 5.1.1). The XMM-Newton spectrum is 
Table A1. A list of all the new XMM-Newton observations considered in this work. The columns of the table report the XMM-Newton OBSID, the $X M M$-Newton revolution, the observation start date and time, the observation duration and the EPIC-PN exposure time after cleaning, the source state $(\mathrm{H}=$ hard state; $\mathrm{S}=$ soft state). The following columns give the 3-6, 6-10 and 8-10 keV observed (absorbed) fluxes in units of $10^{-12} \mathrm{erg} \mathrm{cm}^{-2} \mathrm{~s}^{-1}$. The last column shows, in order, the count rate thresholds applied to select bursting, eclipsing and intense dipping periods, the hard and soft count rates and the threshold to select out intense particle activity periods. A more exhaustive description of the data reduction and cleaning is provided in Section 2.1. With bold characters are reported the XMM-Newton observations simultaneous with the NuSTAR ones. Italic characters indicate XMM-Newton observations corrupted by very bright X-ray transients.

\begin{tabular}{|c|c|c|c|c|c|c|c|c|c|c|}
\hline $\begin{array}{l}\text { XMM-Newton } \\
\text { OBSID }\end{array}$ & & $\operatorname{Rev}$ & START (UTC) & $\begin{array}{l}\text { EXP } \\
(\mathrm{ks})\end{array}$ & $\begin{array}{l}\text { CL EXP } \\
(\mathrm{ks})\end{array}$ & STATE & $\begin{array}{l}F_{3-6} \\
\quad\left(10^{-12}\right.\end{array}$ & $\begin{array}{l}F_{6-10} \\
\mathrm{erg} \mathrm{cm}^{-2}\end{array}$ & $\begin{array}{l}F_{8-10} \\
\left.\mathrm{~s}^{-1}\right)\end{array}$ & $\begin{array}{l}\text { Threshold } \\
\left(\text { ct s}^{-1} \text { ) }\right.\end{array}$ \\
\hline 0790180401 & & 2970 & 2016-02-26 16:20:13 & 37.0 & 28.4 & S & 66.8 & 71.5 & 27.1 & $11 / 1.5 / 1.2 / 4.0 / 3.5 / 18$ \\
\hline 0743630901 & A & 2804 & 2015-04-02 08:59:19 & 36.6 & 4.5 & $S$ & 101.9 & 136.6 & 53.3 & $13 / 1.5 / 1.4 / 7.0 / 5.0 / 1.5$ \\
\hline 0743630801 & $\mathrm{~B}$ & 2804 & 2015-04-01 08:34:19 & 27.0 & 20.1 & $\mathrm{~S}$ & 96.3 & 121.7 & 46.4 & $13 / 1.5 / 1.3 / 6.2 / 4.9 / 1.1$ \\
\hline 0743630601 & $\mathrm{C}$ & 2787 & 2015-02-26 06:18:16 & 33.4 & 21.3 & $\mathrm{~S}$ & 88.0 & 101.7 & 37.7 & 12/1.5/1.4/5.9/4.4/5.0 \\
\hline 0743630501 & $\mathrm{D}$ & 2711 & 2014-09-28 21:01:46 & 40.8 & 32.2 & $\mathrm{H}$ & 7.1 & 10.6 & 4.7 & $4.0 / 0.3 / 1.7 / 0.8 / 0.5 / 1.3$ \\
\hline 0743630401 & $\mathrm{E}$ & 2711 & 2014-09-27 17:30:23 & 34.5 & 18.6 & $\mathrm{H}$ & 7.3 & 10.5 & 4.6 & $3.5 / 0.2 / 1.5 / 0.8 / 0.5 / 2$ \\
\hline 0743630301 & $\mathrm{~F}$ & 2697 & 2014-08-31 20:23:30 & 28.0 & 20.9 & $\mathrm{H}$ & 6.7 & 10.0 & 4.5 & $3.2 / 0.3 / 1.4 / 0.7 / 0.5 / 1.0$ \\
\hline 0743630201 & G & 2697 & 2014-08-30 19:20:01 & 35.0 & 22.4 & $\mathrm{H}$ & 7.5 & 10.7 & 4.7 & $3.5 / 0.3 / 1.4 / 0.75 / 0.55 / 1.5$ \\
\hline 0723410501 & & 2621 & 2014-04-02 03:00:50 & 62.9 & 39.1 & $\mathrm{H}$ & 5.0 & 8.2 & 3.7 & $3.4 / 0.25 / 1.4 / 0.6 / 0.45 / 1.5$ \\
\hline 0723410401 & & 2610 & 2014-03-10 14:10:43 & 57.0 & 38.5 & $\mathrm{H}$ & 5.3 & 8.3 & 3.6 & $3.2 / 0.25 / 1.4 / 0.58 / 0.4 / 0.8$ \\
\hline 0723410301 & & 2605 & 2014-02-28 17:41:27 & 55.0 & 36.0 & $\mathrm{H}$ & 8.9 & 13.9 & 6.2 & $3.5 / 0.3 / 1.4 / 0.85 / 0.6 / 2.0$ \\
\hline
\end{tabular}

Table A2. A list of all NUSTAR observations considered in this work. The columns of the table report the oBSID, the observation start date, the observation duration and cleaned exposure, the source state $(\mathrm{H}=$ hard state; $\mathrm{S}=$ soft state). The following columns give the 3-6, 6-10 and 8-10 keV observed (absorbed) fluxes in units of $10^{-12} \mathrm{erg} \mathrm{cm}^{-2} \mathrm{~s}^{-1}$. The last column shows the count rate thresholds applied to remove bursts, eclipses and dips. With bold characters are reported the observations simultaneous with the XMM-Newton ones. Italic characters indicate observations corrupted by very bright X-ray transients.

\begin{tabular}{|c|c|c|c|c|c|c|c|c|c|}
\hline \multicolumn{2}{|l|}{$\begin{array}{l}\text { NuSTAR } \\
\text { OBSID }\end{array}$} & START (UTC) & $\begin{array}{l}\text { EXP } \\
(\mathrm{ks})\end{array}$ & \multirow[t]{2}{*}{$\begin{array}{l}\text { CL EXP } \\
(\mathrm{ks})\end{array}$} & STATE & \multirow[t]{2}{*}{$F_{3-6}$} & $F_{6-10}$ & $F_{8-10}$ & $\begin{array}{c}\text { Threshold } \\
\left(\mathrm{ct} \mathrm{s}^{-1}\right)\end{array}$ \\
\hline 90101022002 & & 2016-02-18 22:26:08 & 33.8 & & $\mathrm{~S}$ & & & & 5.51 .0 \\
\hline 90101012002 & & 2015-08-11 22:51:08 & 45.1 & 40.4 & $\mathrm{~S}$ & 84.5 & 89.6 & 30.8 & 6.51 .1 \\
\hline 30002002012 & A & 2015-04-02 08:21:07 & 13.2 & 12.1 & S & 90.4 & 116.4 & 45.3 & 6.51 .3 \\
\hline 30002002010 & B & 2015-04-01 06:31:07 & 14.4 & 11.9 & S & 80.8 & 90.6 & 33.7 & 5.01 .2 \\
\hline 30002002008 & & 2015-03-31 04:41:07 & 25.7 & 21.8 & $S$ & 73.6 & 77.3 & 27.9 & 5.00 .9 \\
\hline 30002002006 & $\mathrm{C}$ & $2015-02-25$ 23:41:07 & 29.6 & 26.2 & $S$ & 72.8 & 76.4 & 28.1 & 5.00 .9 \\
\hline 30002002004 & $\mathrm{DE}$ & 2014-09-27 17:31:07 & 67.8 & 59.5 & $\mathrm{H}$ & 4.9 & 7.9 & 3.6 & 2.10 .07 \\
\hline 30002002002 & FG & 2014-08-30 19:46:07 & 60.3 & 50.6 & $\mathrm{H}$ & 4.8 & 7.7 & 3.6 & 2.10 .09 \\
\hline 30001002010 & & 2014-07-04 10:36:07 & 61.5 & 54.7 & $\mathrm{H}$ & 8.5 & 12.4 & 5.5 & 2.10 .14 \\
\hline 30001002008 & & 2014-06-18 02:21:07 & 33.2 & 29.4 & $\mathrm{H}$ & 8.9 & 13.6 & 6.1 & 2.10 .16 \\
\hline 80002013026 & & 2013-08-23 15:41:07 & 32.0 & 26.6 & $\mathrm{~S}$ & 70.3 & 76.0 & 29.2 & 6.50 .7 \\
\hline 80002013024 & & 2013-08-13 00:06:07 & 11.7 & 9.6 & S & 65.2 & 69.7 & 27.8 & 4.50 .7 \\
\hline 80002013022 & & 2013-08-09 09:01:07 & 11.2 & 10.3 & S & 59.1 & 61.6 & 22.8 & 4.40 .8 \\
\hline 80002013020 & & 2013-08-08 15:01:07 & 12.1 & 11.1 & S & 52.6 & 52.7 & 20.0 & 4.00 .6 \\
\hline 80002013018 & & 2013-07-31 01:56:07 & 22.6 & 20.3 & $\mathrm{H}$ & 14.8 & 20.9 & 9.3 & 2.50 .25 \\
\hline
\end{tabular}

corrected for the effects of dust scattering (see Jin et al. 2017). We fit the XMM-Newton data within the $3-10 \mathrm{keV}$ band and the NuSTAR ones in the $5-40 \mathrm{keV}$ band. This model provides a reasonable fit of the data over the considered energy ranges $\left(\chi^{2}=1454.9\right.$ for 1386 dof).

Fig. A3 shows that the NuSTAR data over the $3-5 \mathrm{keV}$ band sit systematically above the extrapolation of the previously obtained best-fitting model. We correct XMM-Newton for dust scattering effects using SOFTABS, but it is beyond the scope of the paper to do the same for $N U S T A R$, therefore we ignore NuSTAR below $5.5 \mathrm{keV}$, where dust scattering effects are prominent.

\section{A3.2 Hard state}

The black and red spectra in Fig. A4 show the hard-state XMMNewton spectra obtained on 2014 September 28 and 29, respectively, during the longest simultaneous NuSTAR exposure (Tables A1 and A2). The green and blue spectra show the simultaneous FPMA and FPMB spectra. Because during the hard state there is no evidence for ionized absorption or a reflection component, we fit these hard-state spectra with a simple power law component absorbed by neutral material.The photon index observed by NUSTAR and within the two XMM-Newton spectra are all consistent with each other 


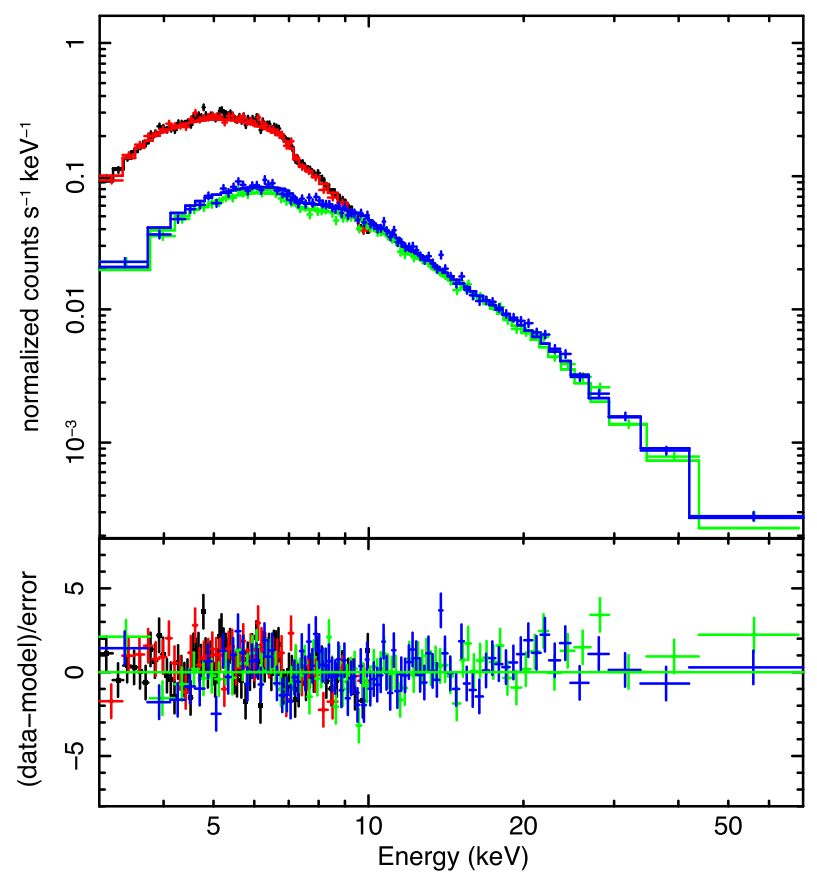

Figure A4. Top panel:The black, red, green and blue data show the two XMM-Newton, NuSTAR FPMA and FPMB spectra obtained during the hard state simultaneous observation with the longest exposure (Tables A1 and A2). The XMM-Newton and NuSTAR spectra are fitted over the 3-10 and 5-40 keV energy band. No residuals are observed at low energy when such model (best fitting the XMM-Newton data) is extrapolated in the 3$5 \mathrm{keV}$ range. Bottom panel: Residuals after fitting the spectra with model DBB-NTH-RR. (although the one measured by NUSTAR is slightly flatter), therefore we required the photon index to be the same for all spectra. We obtained a reasonable fit of the data $\left(\chi^{2}=2255.1\right.$ for 2118 dof $)$. Fig. A4 shows that this model reproduces not only the XMM-Newton but also the NUSTAR data even in the soft X-ray band, leaving no visible residuals.

The small low energy mismatch detectable during the high-flux soft-state observations is not visible during the hard-state observations, indicating that both XMM-Newton and NUSTAR are very well cross-calibrated.

This paper has been typeset from a $\mathrm{T}_{\mathrm{E}} \mathrm{X} / \mathrm{LAT} \mathrm{E} \mathrm{X}$ file prepared by the author. 